\title{
Correlation Between Intensity and Recovery in Credit Risk Models
}

\author{
Raquel M. Gaspar, \\ Department of Finance, \\ Stockholm School of Economics, \\ Box 6501, \\ SE-113 83 Stockholm, \\ SWEDEN \\ e-mail: raquel.gaspar@hhs.se
}

\author{
Irina Slinko, \\ Department of Finance, \\ Stockholm School of Economics, \\ Box 6501, \\ SE-113 83 Stockholm, \\ SWEDEN \\ e-mail: irina.slinko@hhs.se
}

\section{SSE/EFI Working paper Series in Economics and Finance No. 614}

November 2005

\begin{abstract}
We start by presenting a reduced-form multiple default type of model and derive abstract results on the influence of a state variable $X$ on credit spreads, when both the intensity and the loss quota distribution are driven by $X$. The aim is to apply the results to a concrete real life situation, namely, to the influence of macroeconomic risks on credit spreads term structures.

There has been increasing support in the empirical literature that both the probability of default (PD) and the loss given default (LGD) are correlated and driven by macroeconomic variables. Paradoxically, there has been very little effort from the theoretical literature to develop credit risk models that would include this possibility. A possible justification has to do with the increase in complexity this leads to, even for the "treatable" default intensity models.

The goal of this paper is to develop the theoretical framework needed to handle this situation and, through numerical simulation, understand the impact on credit risk term structures of the macroeconomic risks. In the proposed model the state of the economy is modeled trough the dynamics of a market index, that enters directly on the functional form of both the intensity of default $\lambda$ and the distribution of the loss quota $q$ given default. Given this setup, we are able to make periods of economic depression, periods of higher default intensity as well as periods where low recovery is more likely, producing a business cycle effect. Furthermore, we allow for the possibility of an index volatility that depends negatively on the index level and show that, when we include this realistic feature, the impacts on the credit spread term structure are emphasized.
\end{abstract}




\section{Contents}

1 Introduction $\quad 3$

2 The Setup and Abstract Results 4

2.1 Default-free bond market . . . . . . . . . . . . . . . . . 4

2.2 Defaultable bonds market . . . . . . . . . . . . . . . . . 5

2.2 .1 Concepts and Assumptions . . . . . . . . . . . . . . 5

2.2.2 Existence of intensity . . . . . . . . . . . . . . . 7

2.3 Abstract results . . . . . . . . . . . . . . . . . 11

2.4 On the market price of jump risk . . . . . . . . . . . . . . . . 15

3 The Macroeconomic Risks $\quad 17$

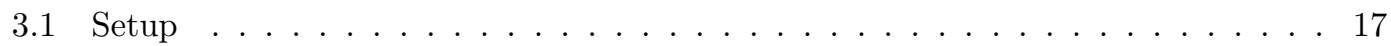

3.2 Credit spreads term structures . . . . . . . . . . . . . . . . 20

4 A concrete model $\quad 22$

4.1 Choosing the market index volatility $\gamma \ldots \ldots \ldots \ldots$

4.2 Choosing default intensities and the loss quota distribution . . . . . . . . . 23

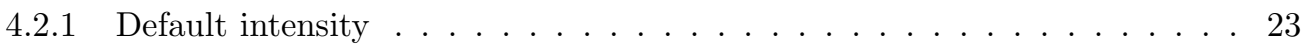

$4.2 .2 \operatorname{Loss}$ quota $q \ldots \ldots \ldots \ldots \ldots$

4.3 Simulation Results . . . . . . . . . . . . . . . . . . . 26

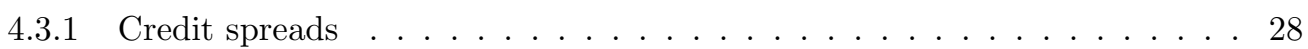

4.3.2 Prices of credit securities . . . . . . . . . . . . . 30

4.4 Additional Considerations . . . . . . . . . . . . . . . . . . . . . 34

4.4 Higher maturities . . . . . . . . . . . . . . . . . . 34

4.4.2 Ratings and different sensitivities . . . . . . . . . . . . . 36

4.4.3 Using implied ATM volatilities as credit spread trackers . . . . . . . . 36

5 Conclusions and future research $\quad 42$ 


\section{Introduction}

Recent empirical studies show that there is a significant systematic risk component in defaultable credit spreads. See Frye (2000a), Frye (2000b), Frye (2003), Altman, Resti, and Sironi (2004), Düllmann and Trapp (2000) or Elton and Gruber (2004). The model underlying the Basel II internal ratings-based capital calculation (see Basel Committee (2003) and Wilde (2001) measures credit portfolio losses only, that is, portfolio losses that are due to external influences and hence cannot be diversified away. This gives us an illustration of what are the main concerns in practice and point out the need of a realistic model for systematic risk. The purpose of this study is to present a reduced-form multiple default model ${ }^{1}$, that analyzes the influence of macroeconomic risks on the credit spread's term structure at the firm level.

The current theoretical literature considers models where only the default intensity, or equivalently, the probability of default (PD) is dependent on a state variable assuming that the loss given default (LGD) is either fixed or at least independent of default intensities. See Wilson (1997), Saunders (1999), JP Morgan (1997), Gordy (2000), or Schönbucher (2001). We take that analysis one step ahead and consider the situation in which the same state variable influences both PD and LGD, making these two quantities dependent of one another.

Both PD and LGD are key in accessing expected capital losses and measuring the exposure of portfolios of defaultable instruments to credit risk. In accessing capital at risk it is of extreme importance not to ignore the interdependence between PD and LGD, since this would lead to underestimation of the true risk beard by portfolio holders.

Eventually, we are interested in the case where our state variable is a representation of macroeconomic risks. As a proxy of the macroeconomic condition we consider a market index. It is well known that market uncertainty and its level are negatively correlated. See, for instance, Gaspar (2001) and a recent study by Giese (2005). That is, periods of recession (low index level) tend to be also periods of high uncertainty (high index volatility) reflecting some sort of market panic, while periods of economic boom are perceived as safe periods and, thus, have low uncertainty. In setting up the dynamics of the market index we incorporate this realistic feature by allowing the index local volatility to depend negatively on the index level.

In terms of the PD and LGD, we concretely take the default intensity and the recovery (given default) to depend on the market situation (the index level). With the PD dependence we try to account for the fact that under bad economic periods it is reasonable to expect more defaults, while with the LGD dependence we try to account for the fact that if the entire market is down, the market value of any firms assets should be lower, and debt holders should recover less if a default occurs.

The main contributions of this study can be summarized as follows.

- We derive abstract results for a multiple default reduced-form model when the default events are modeled by a doubly stochastic marked point process (DSMPP), where both intensity and the marks density depend on a state variable $X$.

- We propose a model for the influence of macroeconomic risks on credit spreads.

- Using a concrete model, we are able to simulate realistic behaviors of credit spreads term structures.

The rest of the paper is organized as follows.

\footnotetext{
${ }^{1}$ For a review of reduced-form models and we refer to Schönbucher (2003).
} 
- In Section 2 we concretize our reduced-form multiple default model and justify the modeling choices, which involve modeling all the needed variables under the risk-neutral measure $\mathbb{Q}$. We start by describing the setup for the default-free and defaultable bond market and we continue by deriving the abstract results. Finally, we discuss the relation between the risk-neutral assumptions and the objective $\mathbb{P}$-assumptions.

- In Section 3 we introduce the macroeconomic model, presenting the index dynamics and justifying the assumptions about its influence of such risks on the intensity and recovery processes using empirical facts. We derive qualitative results on the influence of the market index on credit spreads.

- In Section 4 we give a concrete model for the intensity and recovery dependence and simulate their impacts on credit spread term structures.

- Section 5 concludes, summarizing the main results and pointing out directions for future research.

\section{The Setup and Abstract Results}

We consider a financial market living on a filtered probability space $\left(\Omega, \mathcal{F}, \mathbb{Q},\left(\mathcal{F}_{t}\right)_{0 \leq t \leq T}\right)$ where $\mathbb{Q}$ is the risk-neutral probability measure.

The probability space carries a multidimensional Wiener process $W$ and, in addition, a doubly stochastic marked point process (DSMPP), $\mu(d t, d q)$, on a measurable mark space $(E, \mathcal{E})$ to model the default events.

The filtration $\left(\mathcal{F}_{t}\right)_{0 \leq t \leq T}$ is generated by $W$ and $\mu$, i.e. $\mathcal{F}_{t}=\mathcal{F}_{t}^{W} \vee \mathcal{F}_{t}^{\mu}$.

We, now, introduce the details.

\subsection{Default-free bond market}

We assume the existence of a liquid market for default-free zero-coupon bonds, for every possible maturity $T$. We denote the price at time $t$ of a default-free zero-coupon bond with maturity $T$ by $p(t, T)$.

In order to describe the default-free bonds market we use Heath-Jarrow-Morton (HJM) framework, modeling directly, under the martingale measure $\mathbb{Q}$, the dynamics of the instantaneous forward rates, $f(t, T)$. There is a one-to-one correspondence between zero-coupon bond prices and forward rates, so by assuming existence of zero-coupon bond prices for all maturities we also guarantee existence of forward rates for all maturities. We recall the fundamental relation:

$$
f(t, T)=-\frac{\partial \ln p(t, T)}{\partial T} \quad \Leftrightarrow \quad p(t, T)=\exp \left\{-\int_{t}^{T} f(t, s) d s\right\} .
$$

\section{Assumption 2.1. (Default-free forward rates)}

The dynamics of default free forward rates, under the martingale measure $\mathbb{Q}$, are given by

$$
d f(t, T)=\alpha(t, T) d t+\sigma(t, T) d W_{t},
$$


with

$$
\alpha(t, T)=\sigma(t, T) \int_{t}^{T} \sigma^{*}(t, s) d s
$$

where $\sigma(\cdot, T)$ is a row vector of regular enough adapted processes, $W$ is a $\mathbb{Q}$-Wiener process.

The default free short-rate is $r(t)=f(t, T)$ and the default free zero-coupon bond prices are denoted by $p(t, T)$. No arbitrage and the fundamental relation in (1) give use the bond prices dynamics as

$$
\frac{d p(t, T)}{p(t, T)}=r(t) d t+\eta(t, T) d W(t)
$$

where $\eta(t, T)=-\int_{t}^{T} \sigma(t, s) d s$.

\subsection{Defaultable bonds market}

In addition to the risk-free bond market mentioned above, we consider a defaultable bond market. We assume that each company on the market issues a continuum of bonds with maturities $T$.

Assumptions 2.6 below characterize the default events and the dependence of both the default intensity and the recovery rate distribution on an abstract stochastic state variable $X$.

Assumption 2.2. There exist an underlying stochastic state variable $X$, whose dynamics under the risk neutral measure $\mathbb{Q}$ are given by

$$
d X_{t}=\alpha_{X}\left(t, X_{t}\right) d t+\sigma_{X}\left(t, X_{t}\right) d W_{t}
$$

where $W$, is the same as in (2). ${ }^{2}$

\subsubsection{Concepts and Assumptions}

In Assumption 2.6 we will define the basic multiple default setup. A multiple default setup is based on the observation that whenever the obligor defaults, the company is not liquidated but instead reorganized. The firm and its claims continue to live and operate. However, the face value of the claims is reduced by a fraction $q$. Behind this model is the intuition that, given a distress situation for the obligator's business, the debt holders are willing to accept the renegotiation of their claims (accepting to lose some fraction $q$ of the face value of the claims) in order to avoid a process of bankruptcy, which is typically costly, and allowing the firm to continue operating. ${ }^{3}$ It is possible that a whole sequence of defaults is taking place, every time the company reducing the face value of the debt and the bondholder accepting the conditions of the deal.

Definition 2.3. The loss quota is the fraction by which the promised final payoff of the defaultable claim is reduced at each time of default. We denote the loss quota by $q$.

\footnotetext{
${ }^{2}$ This way we allow for the possibility that one of our factors may be related to the risk-free side of the economy. In that case, some consistency relation must hold between (2) and (4).

${ }^{3}$ This model mimics the effect of a rescue plan as it is described in many bankruptcy codes. The old claimants have to give up some of their claims in order to allow for rescue capital to be invested in the defaulted firm. They are not paid out in cash (this would drain the defaulted firm of valuable liquidity) but in new defaultable bonds of the same maturity. [Schönbucher (2003)]
} 
Definition 2.4. The remaining value, after all reductions in the face value of the defaultable claim due to defaults in the time interval $[0, t]$, is denoted $V(t)$.

Definition 2.5. $\bar{p}(t, T)$ is, at time $t$, the price of a defaultable zero-coupon bond with maturity $T$. The payoff at time $T$ of the bond is, thus, $V(T)$ the remaining part of the face value of the bond after all reductions due to defaults in the time interval $[0, T]$, i.e.,

$$
\bar{p}(T, T)=V(T) .
$$

\section{Assumption 2.6.}

1. We assume that default happens at the following sequence of the stopping times $\tau_{1}<\tau_{2}<$ $\ldots$, where $\tau_{i}$ is the time of the $i$-th jump of our point process.

2. At each default time $\tau_{i}$ the jump size, $q_{i}$ mark or loss quota, is drawn from the mark space $E=(0,1)$.

3. We assume that there is no total loss at default, i.e., the loss quota $q_{i}<1$ for all $i=$ $1,2, \cdots$.

4. We assume that both:

(i) the arrivals of default times $\left(\tau_{i}\right)_{i \geq 1}$

(ii) the distribution of the loss quotas given default $\left(q_{i}\right)_{i \geq 1}$

depend upon our stochastic state process $X$.

Given that at each default time $\tau_{i}$ the final claim amount is reduced by a loss quota $q_{i}$ to $\left(1-q_{i}\right)$ times what it was before, we obtain

$$
V(t)=\prod_{\tau_{i} \leq t}\left(1-q_{i}\right),
$$

where $q_{i}$ is the stochastic marker to the default time $\tau_{i}$.

According to risk-neutral valuation, the price at time $t$, of the defaultable bond with maturity $T$ equals to

$$
\bar{p}(t, T)=\mathbb{E}^{\mathbb{Q}}\left[e^{-\int_{t}^{T} r_{s} d s} V(T) \mid \mathcal{F}_{t}\right]
$$

or, equivalently,

$$
\bar{p}(t, T)=p(t, T) \mathbb{E}^{T}\left[V(T) \mid \mathcal{F}_{t}\right],
$$

where $\mathbb{E}^{T}\left[\cdot \mid \mathcal{F}_{t}\right]$ denote conditional expectation under the $T$-forward measure.

Definition 2.7. We define the instantaneous defaultable forward rate, $\bar{f}(t, T)$, similarly to its risk-free equivalent

$$
\bar{f}(t, T)=-\frac{\partial}{\partial T} \ln \bar{p}(t, T)
$$

The defaultable short rate is defined as $\bar{r}(t)=\bar{f}(t, t)$. 
Using the above definition we also have

$$
\bar{p}(t, T)=V(t) \exp \left\{-\int_{t}^{T} \bar{f}(t, s) d s\right\},
$$

where recall that $\bar{p}(t, t)=V(t)$ and $V(t)$ is given in (5).

Definition 2.8. The short credit spread $s(t)$ is defined as the difference between the defaultable and non-defaultable short rates

$$
s(t)=\bar{r}(t)-r(t) .
$$

Definition 2.9. The forward credit spread $s(t, T)$ is defined as the difference between the defaultable short rate and non-defaultable forward rates

$$
s(t, T)=\bar{f}(t, T)-f(t, T) .
$$

\subsubsection{Existence of intensity}

We start by giving the abstract definitions of a Marked Poisson Point Process, a Cox Process and our Doubly Stochastic Marked Poisson Process (DSMPP). To introduce the definitions we need first to define the following filtrations.

\section{Notation 1. (Filtrations)}

- We call the filtration generated by $W(t)$ the background filtration $\left(\mathcal{F}_{t}^{W}\right)_{t \geq 0}$, and

$$
\mathcal{G}^{W}=\bigvee_{t \geq 0} \mathcal{F}_{t}^{W}
$$

is the information set containing all future and past background information.

In our setup it will be assumed that all the default-free processes are adapted to $\left(\mathcal{F}_{t}^{W}\right)_{t \geq 0}$.

- The full filtration is reached by combining $\left(\mathcal{F}_{t}^{W}\right)_{t \geq 0}$ and the filtration $\left(\mathcal{F}_{t}^{\mu}\right)_{t \geq 0}$ which is generated by MPP $\mu$

$$
\mathcal{F}_{t}=\mathcal{F}_{t}^{W} \vee \mathcal{F}_{t}^{\mu} \text {. }
$$

- We define the filtration generated by all the information concerning the background process $X$, and our only past information on our MPP $\mu$

$$
\mathcal{G}_{t}^{W}=\mathcal{G}^{W} \vee \mathcal{F}_{t}^{\mu}
$$

\section{Definition 2.10. (DSMPP)}

- We call the Marked Point Process $\hat{\mu}$ an $\mathcal{F}_{t}^{\mu}$ - Marked Poisson Process if there exists a deterministic measure $\hat{\nu}$ on $\mathbb{R}_{+} \times E$ such that

$$
\mathbb{P}\left(\hat{\mu}((s, t] \times B)=k \mid \mathcal{F}_{s}^{\mu}\right)=\frac{(\hat{\nu}((s, t] \times B))^{k}}{k !} e^{-\hat{\nu}((s, t] \times B)}, \quad \text { a.s. }, \quad B \in E .
$$


- We call a counting process $N=\left(T_{n}\right)$ adapted to right-continuous filtration a $\mathcal{G}_{t}^{W}$ - Cox Process if there is an $\mathcal{G}^{W}$-measurable random measure $\nu$ satisfying

$$
\mathbb{P}\left(N(s, t]=k \mid \mathcal{G}_{s}^{W}\right)=\frac{(\nu((s, t]))^{k}}{k !} e^{-\nu((s, t])}, \quad \text { a.s } \quad k \in \mathbb{N} .
$$

- We call the Marked Point Process $\mu$ an $\mathcal{G}_{t}^{W}$ - DSMPP if there exists a $\mathcal{G}^{W}$-measurable random measure $\nu$ on $\mathbb{R}_{+} \times E$ such that

$$
\mathbb{P}\left(\mu((s, t] \times B)=k \mid \mathcal{G}_{s}^{W}\right)=\frac{(\nu((s, t] \times B))^{k}}{k !} e^{-\nu((s, t] \times B)}, \quad \text { a.s., } \quad B \in E .
$$

Our goal is to construct a Marked Point Process such that its compensator is allowed to depend on our stochastic state variable $X$ (which in general means that its conditional default distribution as well as intensity are both allowed to depend on $X_{t}$ ), and conditional on the realization of the state variable it is $\mathcal{G}_{t}^{W}$-DSMPP. In other words, we want to prove the existence of MPP such that its compensator $\nu$ is allowed to depended on our stochastic state variable $X$, so we can write

$$
\nu(d t, d q, \omega)=\nu\left(t, d q, X_{t}\right), \quad \mathbb{Q}-\text { a.s. }
$$

We define the compensated point process $\tilde{\mu}(d t, d q)$ as

$$
\tilde{\mu}(d t, d q)=\mu(d t, d q)-\nu\left(t, d q, X_{t}\right) .
$$

The following Theorem shows that the DSMPP with compensator of the form (9) exists.

\section{Theorem 2.11.}

Assume that $\nu$ admits intensity and define $\nu\left(d t, d q, X_{t}\right)=M_{t}\left(d q, X_{t}\right) d t, \mathbb{Q}$-a.s where $M_{t}(d q, x)$ is a deterministic measure on $E$ for any fixed $x$ and $t$.

Let $\hat{\nu}(d t, d q)=m_{t}(d q) d t$ be a deterministic compensator for some Marked Poisson Process $\hat{\mu}$. Assume that:

(i) $M(t, d q, x)$ is measurable w.r.t. $\mathcal{G}^{W}$

(ii) $M(t, d q, x)$ is absolutely continuous w.r.t. $m(t, d q)$ on $\mathcal{E}$, that is,

$$
M_{t}(d q, x)<<m_{t}(d q)
$$

Then, there exists a $\mathcal{G}_{t}^{W}-D S M P P \mu$, such that its compensator is of the form (9).

Proof. We fix $\left(\Omega, \mathcal{F}, \mathbb{P},\left(\mathcal{F}_{t}\right)_{0 \leq t \leq T}\right)$ and a Marked Point Process $\mu$ with the compensator

$$
\hat{\nu}(d t, d q)=m_{t}(d q) d t
$$

and, as before, $\mathcal{G}_{t}^{W}=\mathcal{G}^{W} \vee \mathcal{F}_{t}^{\mu}$.

Since $M_{t}(x, d q)$ is absolutely continuous w.r.t $m_{t}(d q)$ on $\mathcal{E}$, then according to the RadonNikodym Theorem for every $t$ there exists a $\mathcal{E} \times \mathcal{G}^{W}$-measurable nonnegative function $\varphi_{t}(q, x)$, $\varphi: E \times R_{+} \rightarrow R_{+}$, such that

$$
M(t, x, A)=\int_{A} \varphi(t, q, x) m(t, d q), \quad \text { for all } \quad A \in \mathcal{E}
$$


or

$$
M(t, x, d q)=\varphi(t, q, x) m(t, d q)
$$

We define the process $L_{t}$ as

$$
\left\{\begin{aligned}
d L_{t} & =L_{t-} \int_{E}\left\{\varphi\left(t, q, X_{t}\right)-1\right\}\left\{\hat{\mu}(d t, d q)-m_{t}(d q) d t\right\} \\
L_{0} & =1
\end{aligned}\right.
$$

We notice that $\varphi\left(t, q, X_{t}\right) \in \mathcal{G}_{0}^{W}$. Define the new measure on $\mathcal{G}_{t}^{W}, 0 \leq t \leq T$ as

$$
d \mathbb{Q}=L_{t} d \mathbb{P}
$$

According to the Girsanov transformation the $\mathbb{Q}$-compensator of the new process is exactly

$$
\nu(d t, d q)=\hat{\nu}(d t, d q)\left(1+\varphi_{t}\left(q, X_{t}\right)-1\right)=\varphi_{t}\left(q, X_{t}\right) m_{t}(d q) d t=M_{t}\left(d q, X_{t}\right) d t
$$

First, we would like to show that the $\mathbb{Q}$-distribution of $\nu$ is the same as the $\mathbb{P}$-distribution. We note that $\mathcal{G}_{0}^{W}=\mathcal{G}^{W}$ and that

$$
\left.\frac{d \mathbb{Q}}{d \mathbb{P}}\right|_{\mathcal{G}_{0}^{W}}=L_{0}=1
$$

thus, $\mathbb{P}=\mathbb{Q}$ on $\mathcal{G}_{0}^{W}$.

Second, we would like to show that

$$
\mathbb{P}\left(\mu((s, t] \times B)=k \mid \mathcal{G}_{s}^{W}\right)=\frac{(\nu((s, t] \times B))^{k}}{k !} e^{-\nu((s, t] \times B)}, \quad \text { a.s., } \quad B \in E .
$$

We prove (10) using characteristic functions. Define the stochastic process

$$
Y_{t}=\int_{0}^{t} \int_{E} q \hat{\mu}(d t, d q)
$$

Changing the measure we obtain that

$$
\mathbb{E}^{\mathbb{Q}}\left[e^{i u Y_{t}} \mid \mathcal{G}_{0}^{W}\right]=\mathbb{E}^{\mathbb{P}}\left[L_{t} e^{i u Y_{t}} \mid \mathcal{G}_{0}^{W}\right] .
$$

Define $Z_{t}=L_{t} e^{i u Y_{t}}$, then the dynamics of $Z_{t}$ is

$$
\begin{aligned}
d Z_{t}= & L_{t} \int_{E}\left\{e^{i u\left(Y_{t-}+q\right)}-e^{i u Y_{t-}}\right\} \mu(d t, d q) \\
& +L_{t-} e^{i u Y_{t}} \int_{E}\left(\varphi\left(t, q, X_{t}\right)-1\right)\left\{\hat{\mu}(d t, d q)-m_{t}(d q) d t\right\} \\
& +\int_{E} L_{t-}\left(\varphi\left(t, q, X_{t}\right)-1\right)\left\{e^{i u\left(Y_{t-}+q\right)}-e^{i u Y_{t-}}\right\} \hat{\mu}(d t, d q) \\
= & \int_{E} Z_{t-} \varphi\left(t, q, X_{t}\right) m_{t}(d q)\left(e^{i u q}-1\right) d t+\int_{E} Z_{t-}\left(e^{i u q}-1\right) \varphi\left(t, q, X_{t}\right) \tilde{\mu}(d t, d q) \\
& +\int_{E} Z_{t-}\left(\varphi\left(t, q, X_{t}\right)-1\right) \tilde{\mu}(d t, d q)
\end{aligned}
$$

where $\tilde{\mu}=\hat{\mu}(d t, d q)-m_{t}(d q)$. 
We notice also that $Z_{0}=1$, then

$$
\begin{aligned}
Z_{t} & =1+\int_{0}^{t} \int_{E} Z_{s-\varphi}\left(s, q, X_{s}\right) m_{t}(d q)\left(e^{i u q}-1\right) d s+\int_{0}^{t} \ldots \tilde{\mu}(d s, d q) \\
& =1+\int_{0}^{t} \int_{E} Z_{s-}\left(e^{i u q}-1\right) M_{s}\left(d q, X_{s}\right) d s+\int_{0}^{t} \ldots \tilde{\mu}(d s, d q) .
\end{aligned}
$$

Denote $\xi_{t}=\mathbb{E}^{\mathbb{P}}\left[Z_{t} \mid \mathcal{G}_{0}^{W}\right]$, then

$$
\xi_{t}=1+\int_{0}^{t} \int_{E} \xi_{s}\left(e^{i u q}-1\right) M_{s}\left(d q, X_{s}\right) d s .
$$

thus since $\xi_{t}$ does not depend on $q$ and $M_{s}\left(d q, X_{s}\right)$ is $\mathcal{G}_{0}^{W}$-measurable

$$
\xi_{t}=e^{\int_{0}^{t} \int_{E}\left(e^{i u q}-1\right) M_{s}\left(d q, X_{s}\right) d s},
$$

Note that $\nu\left(d t, d q, X_{t}\right)=M_{t}\left(d q, X_{t}\right) d t$ is $\mathcal{G}^{W}$ measurable

The result follows from the fact that the characteristic function of the process

$$
\bar{Y}_{t}=\int_{0}^{t} \int_{E} q \bar{\mu}(d t, d q)
$$

where $\bar{\mu}$ is a Market Poisson Process with compensator $\bar{\nu}(t, d q)$ is given by

$$
\mathbb{E}\left[e^{i u \bar{Y}_{t}}\right]=\exp \left\{\int_{0}^{t} \int_{E}\left(e^{i u q}-1\right) \bar{\nu}(t, d q)\right\} .
$$

In our application we would like to model separately the dependence of the intensity process and of the loss quota distribution on our state variable $X$. This allows us, not only to include empirically observed facts in both quantities but it also makes the interpretation of the results more straightforward. To model the loss quota distribution, in the context of our DSMPP, we need the notion of a stochastic kernel.

Definition 2.12. $K$ is a stochastic kernel from $\mathbb{R}_{+}$to $E$, i.e. from $\left(\mathbb{R}_{+}, \mathcal{B}_{+}\right)$to $(E, \mathcal{E})$, if it is a mapping from $R_{+} \times \mathcal{E}$ into $R_{+}$such that:

- $K(\cdot, A)$ is measurable for all $A \in \mathcal{E}$

- $K(t, \cdot)$ is a measure on $E \forall t$

If $K(t, E)=1$, then the kernel is called a probability distribution.

In our case $K$ will be stochastic only to the extent that $X$ is stochastic. That is, conditional on the state variable realization we will have a deterministic loss quota distribution.

The ideal construction procedure can then be described as follows.

Remark 2.13. Construction procedure We construct our DSMPP $\mu$ as follows.

1. We specify our Wiener driven stochastic state variable $X$. 
2. We specify the intensity $\lambda\left(t, X_{t}\right)$ as a function of our state variable.

3. We specify the instantaneous conditional loss quota distribution as a function of the state variable $K\left(t, d q, X_{t}\right)$.

4. Finally, we construct the stochastic compensator $\nu$

$$
\nu\left(d t, d q, X_{t}\right)=K\left(t, d q, X_{t}\right) \lambda\left(t, X_{t}\right) d t
$$

A compensator representation as in (11) has the additional advantage of satisfying a needed consistency requirement. Indeed, it allows us to value credit derivatives that do not depend on recovery in a consistent way with those that do depend upon recovery. Without the separation result we would have to model at once the whole compensator $M_{t}\left(d q, X_{t}\right)$, which is not very intuitive and we would not know how to derive in a consistent way key ingredients that depend exclusively upon $\lambda\left(t, X_{t}\right)$, like the implied survival probability, the price default digital payoffs or the price defaultable bonds with zero recovery.

Before we go on to the abstract results, we note that Theorem 2.11 suffices to guarantee the existence of a DSMPP $\mu$ with a compensator of the form (11), as we can simply set

$$
M_{t}\left(d q, X_{t}\right)=K\left(t, d q, X_{t}\right) \lambda\left(t, X_{t}\right),
$$

and notice that $M_{t}(d q, x)$ is a measure for fixed $t$ and $x$.

\subsection{Abstract results}

In this section we derive the main results concerning the short and forward credit spreads given the setup above.

Proposition 2.14. Consider a $T$-defaultable claim $\mathcal{X}$. For the purpose of computing expectations, and in particular its price at time $t \leq T$

$$
\mathbb{E}_{t}^{\mathbb{Q}}\left[e^{\int_{t}^{T} r s_{d} s} V(T) \mathcal{X}\right]
$$

it is equivalent to use the following two dynamics for the remaining value process

(i)

$$
\begin{aligned}
\frac{d V(t)}{V(t-)} & =-\int_{0}^{1} q \mu(d t, d q) \\
V(t) & =v
\end{aligned}
$$

(ii)

$$
\begin{aligned}
\frac{d V(t)}{V(t-)} & =-q^{e}\left(t-, X_{t-}\right) d N_{t} \\
V(t) & =v .
\end{aligned}
$$

where $\mu$ is a DSMPP with compensator $\nu\left(t, X_{t}\right)=\lambda\left(t, X_{t}\right) K\left(t, d q, X_{t}\right) d t, N$ is a Cox process with intensity $\lambda\left(t, X_{t}\right)$ and we define

$$
q^{e}\left(t, X_{t}\right)=\int_{0}^{1} K\left(t, d q, X_{t}\right) .
$$


Proof. Using the $V$ dynamics in (i) we get,

$$
\begin{aligned}
\mathbb{E}^{\mathbb{Q}}\left[e^{-\int_{t}^{T} r_{s} d s}\right. & \left.V(T) \mathcal{X} \mid \mathcal{F}_{t}\right]= \\
& =V(t) \underbrace{\mathbb{E}^{\mathbb{Q}}\left[e^{-\int_{t}^{T} r_{s} d s} \mathcal{X} \mid \mathcal{F}_{t}\right]}_{\pi(t, \mathcal{X})}-\mathbb{E}^{\mathbb{Q}}\left[e^{-\int_{t}^{T} r_{s} d s} \int_{t}^{T} \int_{0}^{1} q V_{s-} \mu(d q, d s) \mathcal{X} \mid \mathcal{F}_{t}\right] \\
& =V(t) \pi(t, \mathcal{X})-\mathbb{E}^{\mathbb{Q}}\left[\mathbb{E}^{\mathbb{Q}}\left[e^{-\int_{t}^{T} r_{s} d s} \int_{t}^{T} \int_{0}^{1} q V_{s-} \mu(d q, d s) \mathcal{X} \mid \mathcal{G}_{t}^{W}\right] \mid \mathcal{F}_{t}\right] \\
& =V(t) \pi(t, \mathcal{X})-\mathbb{E}^{\mathbb{Q}}\left[e^{-\int_{t}^{T} r_{s} d s} \mathbb{E}^{\mathbb{Q}}\left[\int_{t}^{T} \int_{0}^{1} q V_{s-} \mu(d q, d s) \mathcal{X} \mid \mathcal{G}_{t}^{W}\right] \mid \mathcal{F}_{t}\right] \\
& =V(t) \pi(t, \mathcal{X})-\mathbb{E}^{\mathbb{Q}}\left[e^{-\int_{t}^{T} r_{s} d s} \int_{t}^{T} V_{s-}\left\{\int_{0}^{1} q K\left(s, d q, X_{s}\right)\right\} \lambda\left(s, X_{s}\right) d s \mathcal{X} \mid \mathcal{F}_{t}\right] \\
& =V(t) \pi(t, \mathcal{X})-\mathbb{E}^{\mathbb{Q}}\left[e^{-\int_{t}^{T} r_{s} d s} \int_{t}^{T} V_{s-} q^{e}\left(s, X_{s}\right) \lambda\left(s, X_{s}\right) d s \mathcal{X} \mid \mathcal{F}_{t}\right]
\end{aligned}
$$

Using the $V$ dynamics in (ii) we get,

$$
\begin{aligned}
\mathbb{E}^{\mathbb{Q}}\left[e^{-\int_{t}^{T} r_{s} d s} V(T) \mathcal{X} \mid \mathcal{F}_{t}\right]= \\
=V(t) \underbrace{\mathbb{E}^{\mathbb{Q}}\left[e^{-\int_{t}^{T} r_{s} d s} \mathcal{X} \mid \mathcal{F}_{t}\right]}_{\pi(t, \mathcal{X})}-\mathbb{E}^{\mathbb{Q}}\left[e^{-\int_{t}^{T} r_{s} d s} \int_{t}^{T} V_{s-} q^{e}\left(s, X_{s}\right) d N(s) \mathcal{X} \mid \mathcal{F}_{t}\right] \\
=V(t) \pi(t, \mathcal{X})-\mathbb{E}^{\mathbb{Q}}\left[\mathbb{E}^{\mathbb{Q}}\left[e^{-\int_{t}^{T} r_{s} d s} \int_{t}^{T} V_{s-} q^{e}\left(s, X_{s}\right) d N(s) \mathcal{X} \mid \mathcal{G}_{t}^{W}\right] \mid \mathcal{F}_{t}\right] \\
=V(t) \pi(t, \mathcal{X})-\mathbb{E}^{\mathbb{Q}}\left[e^{-\int_{t}^{T} r_{s} d s} \mathbb{E}^{\mathbb{Q}}\left[\int_{t}^{T} V_{s-} q^{e}\left(s, X_{s}\right) d N(s) \mathcal{X} \mid \mathcal{G}_{t}^{W}\right] \mid \mathcal{F}_{t}\right] \\
=V(t) \pi(t, \mathcal{X})-\mathbb{E}^{\mathbb{Q}}\left[e^{-\int_{t}^{T} r_{s} d s} \int_{t}^{T} V_{s-} q^{e}\left(s, X_{s}\right) \lambda\left(s, X_{s}\right) d s \mathcal{X} \mid \mathcal{F}_{t}\right]
\end{aligned}
$$

The results follows from comparing the final expressions on both cases.

Proposition 2.15. Given Assumption 2.6, and under the martingale measure $\mathbb{Q}$.

1. The short credit spreads, $s(t)$, have the following functional form

$$
s(t)=\lambda\left(t, X_{t}\right) q^{e}\left(t, X_{t}\right)>0
$$

where

$$
q^{e}\left(t, X_{t}\right)=\int_{0}^{1} q K\left(t, d q, X_{t}\right)
$$

can be interpreted as the locally expected loss quota (which is positive for $q>0$ ). 
2. Then the forward credit spread $s(t, T)$ takes the form

$$
s(t, T)=\frac{\mathbb{E}_{t}^{\mathbb{Q}}\left[\left\{r(T)+\lambda\left(T, X_{T}\right) q^{e}\left(T, X_{T}\right)\right\} e^{-\int_{t}^{T}\left\{r(s)+\lambda\left(s, X_{s}\right) q^{e}\left(s, X_{s}\right)\right\} d s}\right]}{\mathbb{E}_{t}^{\mathbb{Q}}\left[e^{-\int_{t}^{T}\left\{r(s)+\lambda\left(s, X_{s}\right) q^{e}\left(s, X_{s}\right)\right\} d s}\right]}-f(t, T)
$$

Proof. The time $t$ price of the defaultable zero-coupon bond with maturity $T$ is equal to

$$
\bar{p}(t, T)=\mathbb{E}^{\mathbb{Q}}\left[e^{-\int_{t}^{T} r_{s} d s} V(T) \mid \mathcal{F}_{t}\right],
$$

where $V(T)$ is the residual of the face value after multiple defaults up to time $T$.

Making use of Proposition 2.14, instead of $\frac{d V(t)}{V(t-)}=-\int_{0}^{1} q \mu(d t, d q)$ with our DSMPP $\mu$ (these dynamics follow directly from (5)), we use

$$
\frac{d V(t)}{V(t-)}=-q^{e}\left(t-, X_{t-}\right) d N_{t}
$$

where $N$ is the Cox process with intensity $\lambda\left(t, X_{t}\right)$.

For every fixed $t$, define $Z(u)$ as follows

$$
Z(u)=e^{\int_{t}^{u} q^{e}\left(s, X_{s}\right) \lambda\left(s, X_{s}\right) d s} V(u) .
$$

We note that then the dynamics of $Z(u)$ take the form

$$
d Z(u)=-Z_{u-} q^{e}\left(u-, X_{u-}\right)\left\{d N_{u}-\lambda\left(u, X_{u}\right) d u\right\}, \quad u \geq t, \quad t \text {-fixed }
$$

and $Z(u)$ is a $\mathbb{Q}$-martingale conditional on the filtration $\mathcal{F}_{t}^{W}$. Thus,

$$
\mathbb{E}^{\mathbb{Q}}\left[Z(T) \mid \mathcal{F}_{t}^{W}\right]=Z(t) .
$$

The price of a defaultable bond is then can be found as

$$
\begin{aligned}
\bar{p}(t, T) & =\mathbb{E}^{\mathbb{Q}}\left[e^{-\int_{t}^{T} r_{s} d s} V(T) \mid \mathcal{F}_{t}\right]=\mathbb{E}^{\mathbb{Q}}\left[e^{-\int_{t}^{T} r_{s} d s} e^{-\int_{t}^{T} q^{e}\left(s, X_{s}\right) \lambda\left(s, X_{s}\right) d s} Z(T) \mid \mathcal{F}_{t}\right] \\
& =\mathbb{E}^{\mathbb{Q}}\left[\mathbb{E}^{\mathbb{Q}}\left[e^{-\int_{t}^{T} r_{s} d s} e^{-\int_{t}^{T} q^{e}\left(s, X_{s}\right) \lambda\left(s, X_{s}\right) d s} Z(T) \mid \mathcal{G}_{t}^{W}\right] \mid \mathcal{F}_{t}\right] \\
& =\mathbb{E}^{\mathbb{Q}}\left[e^{-\int_{t}^{T} r_{s} d s} e^{-\int_{t}^{T} q^{e}\left(s, X_{s}\right) \lambda\left(s, X_{s}\right) d s} \mathbb{E}^{\mathbb{Q}}\left[Z(T) \mid \mathcal{G}_{t}^{W}\right] \mid \mathcal{F}_{t}\right] \\
& =\mathbb{E}^{\mathbb{Q}}\left[e^{-\int_{t}^{T} r_{s} d s} e^{-\int_{t}^{T} q^{e}\left(s, X_{s}\right) \lambda\left(s, X_{s}\right) d s} Z(t) \mid \mathcal{F}_{t}\right] \\
& =V(t) \mathbb{E}^{\mathbb{Q}}\left[e^{-\int_{t}^{T} r(s) d s} e^{-\int_{t}^{T} q^{e}\left(s, X_{s}\right) \lambda\left(s, X_{s}\right) d s} \mid \mathcal{F}_{t}\right] \\
& =V(t) \mathbb{E}^{\mathbb{Q}}\left[e^{-\int_{t}^{T}\left\{r(s)+q^{e}\left(s, X_{s}\right) \lambda\left(s, X_{s}\right)\right\} d s} \mid \mathcal{F}_{t}\right] .
\end{aligned}
$$

Using the basic relations between defaultable bond prices and defaultable forward rates in (7) we can, thus, write

$$
\bar{f}(t, T)=\frac{\mathbb{E}_{t}^{\mathbb{Q}}\left[\left\{r(T)+\lambda\left(T, X_{T}\right) q^{e}\left(T, X_{T}\right)\right\} e^{-\int_{t}^{T}\left\{r(s)+\lambda\left(s, X_{s}\right) q^{e}\left(s, X_{s}\right)\right\} d s}\right]}{\mathbb{E}_{t}^{\mathbb{Q}}\left[e^{-\int_{t}^{T}\left\{r(s)+\lambda\left(s, X_{s}\right) q^{e}\left(s, X_{s}\right)\right\} d s}\right]} .
$$

Finally using that $\bar{f}(t, t)=\bar{r}(t)$ in the above expression we obtain

$$
\bar{r}\left(t, r_{t}, X_{t}\right)=r(t)+q^{e}\left(t, X_{t}\right) \lambda\left(t, X_{t}\right) .
$$

The result follow from $s(t)=\bar{r}(t)-r(t)$ and $s(t, T)=\bar{f}(t, T)-f(t, T)$. 
From the proof of the previous Proposition results also a nice formulation for the defaultable bond prices.

Corollary 2.16. Under the conditions of Proposition 2.15 we have

$$
\bar{p}(t, T)=V(t) \mathbb{E}_{t}^{\mathbb{Q}}\left[e^{-\int_{t}^{T} \bar{r}_{s} d s}\right] .
$$

It is obvious from (15) that the expression of the forward credit spread looks quite messy. This happens because we choose to present it under the martingale measure $\mathbb{Q}$. The next lemma give us two simpler representations at the cost of using forward measures.

Lemma 2.17. The forward credit spread, $s(t, T)$, has the following representation, equivalent to (15)

(i)

$$
s(t, T)=-\frac{\partial}{\partial T} \ln \left(\mathbb{E}_{t}^{T}[V(T)]\right)
$$

where $\mathbb{E}_{t}^{T}[\cdot]$ stands for expectation under the $T$-forward measure.

(ii)

$$
s(t, T)=\overline{\mathbb{E}}_{t}^{T}[\bar{r}(T)]-\mathbb{E}_{t}^{T}[r(T)] .
$$

where $\overline{\mathbb{E}}_{t}^{T}$ stands for expectation under the measure $\overline{\mathbb{Q}}^{T}$ where we have

$$
L^{T}=\frac{d \overline{\mathbb{Q}}^{T}}{d \mathbb{Q}}=\frac{V(0) e^{-\int_{0}^{T} r_{s}+\lambda\left(s, X_{s}\right) q^{e}\left(s, X_{s}\right) d s}}{\bar{p}(0, T)} \text { on } \mathcal{F}_{T}
$$

Proof. To prove $(i)$ we note that

$$
\begin{aligned}
\bar{p}(t, T) & =\mathbb{E}_{t}^{\mathbb{Q}}\left[e^{-\int_{t}^{T} r(u) d u} V(T)\right] \\
& =p(t, T) \mathbb{E}_{t}^{T}[V(T)]
\end{aligned}
$$

From the definition of the forward defaultable rate and the forward credit spread we also have

$$
\begin{aligned}
\bar{p}(t, T) & =V(t) e^{-\int_{t}^{T} \bar{f}(t, u) d u} \\
& =V(t) e^{-\int_{t}^{T} f(t, u) d u} e^{-\int_{t}^{T} s(t, u) d u}=V(t) p(t, T) e^{-\int_{t}^{T} s(t, u) d u} .
\end{aligned}
$$

Comparing we realize $V(t) e^{-\int_{t}^{T} s(t, u) d u}=\mathbb{E}_{t}^{T}[V(T)]$, differentiating w.r.t. $T$ and solving for $s(t, T)$ we get

$$
s(t, T)=\frac{\partial}{\partial T} \ln \left(\frac{V(t)}{\mathbb{E}_{t}^{T}[V(T)]}\right)=\underbrace{\frac{\partial}{\partial T} \ln (V(t))}_{0}-\frac{\partial}{\partial T} \ln \left(\mathbb{E}_{t}^{T}[V(T)]\right) .
$$

To prove $(i i)$ we start by noting that it is a well known fact that for the risk-free rates $f(t, T)=$ $\mathbb{E}_{t}^{T}[r(T)] .4$

Thus, it remains to show that

$$
\bar{f}(t, T)=\overline{\mathbb{E}}_{t}^{T}[\bar{r}(T)]
$$

${ }^{4}$ See Björk (2004) for further details. 
Using $\bar{r}(t)=r(t)+q^{e}\left(t, X_{t}\right) \lambda\left(t, X_{t}\right)$, equation (17) becomes

$$
\bar{f}(t, T)=\frac{\mathbb{E}_{t}^{\mathbb{Q}}\left[\bar{r}(T) e^{\left.-\int_{t}^{T}\left\{r(s)+\lambda\left(s, X_{s}\right) q^{e}\left(s, X_{s}\right)\right\} d s\right]}\right.}{\mathbb{E}_{t}^{\mathbb{Q}}\left[e^{-\int_{t}^{T}\left\{r(s)+\lambda\left(s, X_{s}\right) q^{e}\left(s, X_{s}\right)\right\} d s}\right]} .
$$

Since $\frac{\bar{p}(t, T)}{V(t)}=\mathbb{E}^{\mathbb{Q}}\left[e^{-\int_{t}^{T}\left\{r(s)+q^{e}\left(s, X_{s}\right) \lambda\left(s, X_{s}\right)\right\} d s} \mid \mathcal{F}_{t}\right]$ we have

$$
\bar{f}(t, T) \frac{\bar{p}(t, T)}{V(t)}=\mathbb{E}_{t}^{\mathbb{Q}}\left[\bar{r}(T) e^{-\int_{t}^{T}\left\{r(s)+\lambda\left(s, X_{s}\right) q^{e}\left(s, X_{s}\right)\right\} d s}\right] .
$$

For the r.h.s the following holds

$$
\begin{aligned}
& \mathbb{E}_{t}^{\mathbb{Q}}\left[\bar{r}(T) e^{\left.-\int_{t}^{T}\left\{r(s)+\lambda\left(s, X_{s}\right) q^{e}\left(s, X_{s}\right)\right\} d s\right]}=\right. \\
&=\mathbb{E}_{t}^{\mathbb{Q}}[\bar{r}(T) \underbrace{\left.\frac{V(0) e^{-\int_{0}^{T}\left\{r(s)+\lambda\left(s, X_{s}\right) q^{e}\left(s, X_{s}\right)\right\} d s}}{\bar{p}(0, T)}\right]}_{L^{T}} \frac{e^{\int_{0}^{t}\left\{r(s)+\lambda\left(s, X_{s}\right) q^{e}\left(s, X_{s}\right)\right\} d s} \bar{p}(0, T)}{V(0)} \\
&= \overline{\mathbb{E}}_{t}^{T} r(T) \mathbb{E}_{t}^{\mathbb{Q}}\left[L^{T}\right] \frac{e^{\int_{0}^{t}\left\{r(s)+\lambda\left(s, X_{s}\right) q^{e}\left(s, X_{s}\right)\right\} d s} \bar{p}(0, T)}{V(0)} \\
&=\overline{\mathbb{E}}_{t}^{T} r(T) \mathbb{E}_{t}^{\mathbb{Q}}\left[e^{\left.-\int_{t}^{T}\left\{r(s)+\lambda\left(s, X_{s}\right) q^{e}\left(s, X_{s}\right)\right\} d s\right]}\right. \\
&=\frac{\bar{p}(t, T)}{V(t)} \overline{\mathbb{E}}_{t}^{T}[r(T)]
\end{aligned}
$$

Where we define $L^{T}=\frac{V(0) e^{-\int_{0}^{T}\left\{r(s)+\lambda\left(s, X_{s}\right) q^{e}\left(s, X_{s}\right)\right\} d s}}{\bar{p}(0, T)}$ on $\mathcal{F}_{T}$.

Comparing with the l.h.s we get $\bar{f}(t, T)=\overline{\mathbb{E}}_{t}^{T} \bar{r}(T)$.

\subsection{On the market price of jump risk}

We now note that the setup has been defined under the martingale measure $\mathbb{Q}$. Now we specify the implicit assumption on the market price of jump risk that will allows us to extrapolate from our objective intuitions ( $\mathbb{P}$-intuitions) when setting up a concrete model later on.

In order to see the connections between the intensities under the different measures we recall the appropriate Girsanov theorem ${ }^{5}$.

\section{Theorem 2.18. (Girsanov for DSMPP)}

Let $(\Omega, \mathcal{F}, \mathbf{F}, \mathbb{Q})$ be a filtered probability space which supports $\mathbb{Q}$-Brownian motion $W(t)$ and a marked point process $\mu(d t, d q)$, where the marker $q$ is drawn from the mark space $X=[0,1]$. The $\mathbb{Q}$-compensator is assumed to take the form $\nu(d t, d q)=K(t, d q) \lambda(t) d t$ under $\mathbb{Q}$. Here $\lambda$ is the $\mathbb{Q}$-intensity of the arrivals of the point process and $K(t, d q)$ is the $\mathbb{Q}$-conditional distribution of the marker. Let $h$ be a predictable process and $\phi_{t}(q), \phi_{t}(q)>-1$ a predictable function with the properties

$$
\int_{0}^{t}\|h\|^{2} d s<\infty, \quad \int_{0}^{t} \int_{X}\left|\phi_{t}(q)\right| K(s, d q) \lambda(s) d s<\infty .
$$

\footnotetext{
${ }^{5}$ See, for example, Björk (2004) or Schönbucher (2003).
} 
Define the process $L(t)$ by

$$
\left\{\begin{array}{rl}
\frac{d L(t)}{L(t-)} & =h(t) d W(t)+\int_{X} \phi(t)\{\mu(d t, d q)-\nu(d t, d q)\} \\
L(0) & =1
\end{array} .\right.
$$

Define the probability measure $\mathbb{P}$ as follows

$$
\frac{d \mathbb{P}}{d \mathbb{Q}}=L(t) \quad \text { on } \quad \mathcal{F}_{t} \quad \forall t>0 .
$$

Then:

1. The process $W_{\mathbb{P}}$ is a Brownian motion and

$$
d W_{P}(t)=d W(t)-h(t) d t .
$$

2. The predictable compensator under $\mathbb{P}$ is as follows

$$
\nu^{P}(d t, d q)=\left(1+\phi_{t}(q)\right) \nu(d t, d q) .
$$

$\phi_{t}(q)$ in (19) is the market price of jump risk and, in principle, is stochastic $\phi_{t}(q, \omega, X)$.

The following Lemma shows that in the case when Girsanov kernels are deterministic the measure transformation does not affect $K$ and gives us the relationship between the intensities of default under $\mathbb{P}$ and under $\mathbb{Q}$.

Lemma 2.19. Assume that the market price of jump risk, $\phi$ in (19) is a deterministic function of time. That is

$$
\phi_{t}(q, \omega)=\phi(t) .
$$

Then,

1. The $\mathbb{Q}$-default intensity, $\lambda$, relates to the $\mathbb{P}$-default intensity $\lambda^{P}$, by

$$
\lambda^{P}(t, r, I)=\lambda(t, r, I)(1+\phi(t))
$$

2. The $\mathbb{Q}$-loss quota distribution, conditional on default, $K_{t}(d q)$, equals to the conditional on default loss quota distribution under $\mathbb{P}, K_{t}^{P}(d q)$.

Proof. The predictable compensator takes the form

$$
\nu(d t, d q)=M_{t}(d q) d t=\lambda(t) K(t, d q) d t
$$

where

$$
\lambda(t)=M_{t}(E), \quad K(t, d q)=\frac{M_{t}(d q)}{M_{t}(E)}
$$

From the Girsanov theorem we obtain that under $\mathbb{P}$

$$
\begin{aligned}
& M_{t}^{P}(d q)=M_{t}(d q)(1+\phi(t)) \\
& \lambda^{P}(t)=\int_{E}(1+\phi(t)) M_{t}(d q)
\end{aligned}
$$


Thus is case if $\phi(t)$ is deterministic and does not depend on $q$ we see that

$$
\begin{aligned}
\lambda_{t}^{P} & =(1+\phi(t)) M_{t}(E)=(1+\phi(t)) \lambda(t) \\
K_{t}^{P}(d q) & =\frac{(1+\phi(t)) M_{t}(d q)}{\int_{E}(1+\phi(t)) M_{t}(d q)}=\frac{M_{t}(d q)}{\int_{E} M_{t}(d q)}=K_{t}(d q) .
\end{aligned}
$$

We see that, in this case, the conditional distribution of the loss quota remains unchanged while intensity changes according to (22), i.e. multiplied by a deterministic function of time. This also means that once we have specified the influence of the state variable $X$ under $\mathbb{Q}$ we have also specified its influence under the objective measure (up to some deterministic factor in the case of the intensity process $\lambda$ ).

Obviously it is discussable if assuming (20) is a very strong restriction or not. For this study we assume it is not so we can use our objective intuitions in setting up the applied model for macroeconomic risks. Nonetheless, the unhappy reader, can also interpret all our results as only $\mathbb{Q}$-results.

\section{The Macroeconomic Risks}

\section{$3.1 \quad$ Setup}

We model the systematic risk of an economy by considering what we call a market index, and we deal with both the situation when this market index is the price of an important traded asset in the economy and when it is not the price of any traded asset.

An example of an index that is the price of a traded asset is, say, oil price. Indices, like a stock market indices or any other type of indices, on the other hand, are examples of indices that are not prices of traded assets. We consider the two possibilities at all times.

It is rather well-known that the market index' volatility (for example S\&P500) seem to increase when the market as a whole is depressed (low values of the index) and, on the contrary, the volatility decreases when the market index is high (see Gaspar (2001)). In order to account for this fact, we make market index volatility dependent on the index level.

\section{Assumption 3.1. (Market Index)}

Under the martingale measure $\mathbb{Q}$, the market index I, satisfies the following stochastic differential equation (SDE)

- When I is the price of a traded asset

$$
d I_{t}=r(t) I_{t} d t+\gamma\left(t, I_{t}\right) I_{t} d W(t)
$$

- When I is not the price of a traded asset

$$
d I_{t}=\zeta(t) I_{t} d t+\gamma\left(t, I_{t}\right) I_{t} d W(t)
$$

where $r$ is the short rate, $\gamma$ is a row-vector, $W$ is a $\mathbb{Q}$-Wiener process.

Furthermore, for each entry $\gamma_{i}$, the following holds 


$$
\frac{\partial \gamma_{i}}{\partial I}(t, I)<0
$$

The property (i) in (24) represents the empirically observed fact that periods when the market as a whole is depressed are periods with higher volatility, while booms are associated with low volatilities.

Since in our setup it would be wrong to consider $I$ independent of the risk-free short rate $r$ (at least under the risk neutral measure $\mathbb{Q}$ ), and we cannot ignore its influence, we can also consider $r$ as one of the factors.

We will now present explicitly the dependence between the default intensities and the loss quota distribution on the factors. The results from the abstract section can be immediately used with $X=\left[\begin{array}{l}I \\ r\end{array}\right]$

Finally, it is also reasonable to assume that firms less sensitive to systematic risks will suffer less in terms of an increase in their default intensities, than firms more sensitive. We, thus, introduce a measure of sensitivity to systematic risk, $\epsilon, \epsilon \in[0,1]$.

Assumption 3.2. (The default Intensity)

The intensity is a deterministic function of $(t, r, I, \epsilon)$. Furthermore, we have

(ii)

$$
\lambda(t, r, I, 0)=\bar{\lambda} \quad \bar{\lambda} \in \mathbb{R}_{+}
$$

(iii)

(iv)

$$
\frac{\partial \lambda(t, r, I, \epsilon)}{\partial \epsilon}>0
$$

$$
\frac{\partial \lambda(t, r, I, \epsilon)}{\partial I}<0
$$

(v)

$$
\frac{\partial \lambda(t, r, I, \epsilon)}{\partial r}>0
$$

Assumption 3.2 is based on the intuition that if a firm's financial situation is strong enough, then it should not really matter if the market is in a boom or in a depression. That is, firms that are financially solid should be much less sensitive to the market situation than firms in less solid financial situation. One can also see the parameter $\epsilon$ as a measure of credit worthiness of a firm. Firms with high credit worthiness typically tend to be less sensitive to the business cycle influence than firms with less credit worthiness.

If this is so, then it makes sense to have properties (ii) and (iii) in (25) and (26), respectively.

Properties (iv) and (v) (eq (27),(28)) tell us that if the default intensity depends upon macroeconomic variables, then the $\mathrm{PD}$ is higher in depressions or periods of high risk-free interest rates, and lower in booms or periods with low risk-free interest rates. The influence of the index value has to do with the increase in uncertainty in depressions, while the influence of the short rate is related to the increase in the difficulty of refinancing existent debt when the cost of borrowing money is higher (if a firm can only borrow money at high costs the PD is higher). 


\section{Assumption 3.3. (Loss Quota)}

The conditional distribution of loss quota is a deterministic function of $(t, r, I) . K$ is a stochastic kernel from $R_{+} \times R_{+} \times R_{+} \rightarrow[0,1]$ for any realization of $(t, r, I)$

We denote the cumulative distribution function of loss quota conditional on default as $\tilde{K}$

$$
\tilde{K}(t, r, I, x)=\int_{0}^{x} K(t, r, I, d q), \quad \int_{0}^{1} K(t, r, I, d q)=1, \quad \forall t, r, I
$$

with the following properties

$$
\begin{array}{rlll}
\tilde{K}\left(t, r, I_{1}, x\right) \geq \tilde{K}\left(t, r, I_{2}, x\right), & \text { if } & I_{1} \geq I_{2}, & \forall x \in R, \\
\tilde{K}\left(t, r_{1}, I, x\right) \leq \tilde{K}\left(t, r_{2}, I, x\right), & \text { if } & r_{1} \geq r_{2}, & \forall x \in R .
\end{array}
$$

That is

(vi)

$$
\frac{\partial \tilde{K}(t, r, I, x)}{\partial I}>0
$$

(vii)

$$
\frac{\partial \tilde{K}(t, r, I, x)}{\partial r} \leq 0
$$

For fixed $(t, r), \tilde{K}(t, r, I, x)$ stochastically dominates all the conditional distributions with parameter $\underline{I}$, such that $\underline{I} \leq I$ and, for fixed $(t, I) \tilde{K}(t, r, I, x)$ stochastically dominates all the conditional distributions with parameter $\bar{r}$, such that $\bar{r} \geq r$.

Property (vi) in (29) can be justified by the following argument. Given that default has occured and the debt holders are negotiating their loss quota so that the firm is able to continue operating (assumption underlying any multi-default model), it seems reasonable that if the firms assets are worth less they are willing to give in more (higher loss quota) since they would not get that much in case of bankruptcy anyway. In addition bankruptcy costs tend to be higher in depression periods, emphasizing this effect.

As to the risk-free interest rate, the argument for property (vii) in (30) is harder to support, so it is our opinion it is reasonable to assume $\frac{\partial \tilde{K}(t, r, I, x)}{\partial r} \leq 0$, which includes the possibility of no influence. However, if we assume that there exists an impact, then we argue that it should be in the direction of higher interest rates leading to a decrease in the probability of lower loss quotas. In high interest rate periods debt holders have better side options to invest there money and are willing to give in less.

From the stochastic dominance assumption above we can now infer the impacts on the expected loss quota.

Lemma 3.4. Given Assumption 3.3, the following relations hold for the expected value

$$
q^{e}(r, I)=\int_{0}^{1} q K(r, I, d q),
$$

(viii)

$$
\frac{\partial q^{e}(r, I)}{\partial I}<0
$$


(ix)

$$
\frac{\partial q^{e}(r, I)}{\partial r} \geq 0
$$

Proof. The proof is similar to both properties and is a consequence of (vi) $\Rightarrow$ (viii) and (vii) $\Rightarrow$ (ix). We show it for the index value case.

$$
q^{e}(r, I)=\int_{0}^{1} q K(r, I, d q)=\int_{0}^{1} q d \tilde{K}(t, r, I, q) .
$$

Integrating by parts we get

$$
\begin{aligned}
\int_{0}^{1} q d \tilde{K}(t, r, I, q) & =\left.q \tilde{K}(t, r, I, q)\right|_{0} ^{1}-\int_{0}^{1} \tilde{K}(t, r, I, q) d q \\
& =q \underbrace{\tilde{K}(t, r, I, 1)}_{1}-q \underbrace{\tilde{K}(t, r, I, 0)}_{0}-\int_{0}^{1} \tilde{K}(t, r, I, q) d q \\
& =q-\int_{0}^{1} \tilde{K}(t, r, I, q) d q .
\end{aligned}
$$

The results follows from differentiating this last expression w.r.t $I$ and using the property $(v i)$

$$
\frac{\partial q^{e}(r, I)}{\partial I}=-\int_{0}^{1} \underbrace{\frac{\partial \tilde{K}(t, r, I, q)}{\partial I}}_{>0, \forall q} d q<0 .
$$

The same argument will work for the interest rate $r$.

\section{Remark 3.5. (Tractability)}

We note that besides the above mentioned properties $K$, conditional on the state variable information, must be the distribution of a random variable taking values in $(0,1)$, and the intensity $\lambda$ must be always positive. It is, thus, extremely hard to find a treatable model where these two facts together with properties (i)-(ix) are satisfied.

In particular, we have found that no model of affine or quadratic spreads ${ }^{6}$ will verify all the above properties.

Given these tractability difficulties we go on with the analysis and draw qualitative results of the influence of the market index on credit spreads.

\section{$3.2 \quad$ Credit spreads term structures}

Using the abstract result on Proposition 2.15 and Assumptions 3.2 and 3.3 we can study the impacts of our macroeconomic variables $X=\left[\begin{array}{l}I \\ r\end{array}\right]$ on the short spread $s(t)$.

Remark 3.6. Given the results in Proposition 2.15, Assumption 3.2 and Lemma 3.4, the short credit spread can be rewritten as a function of $(t, r, I, \epsilon)$ and

$$
s(t, r, I, \epsilon)=\lambda(t, r, I, \epsilon) q^{e}(t, r, I) .
$$

\footnotetext{
${ }^{6}$ Outside the class of affine or quadratic spread models it is basically impossible to find closed-form solutions.
} 
Furthermore we have

$$
\begin{aligned}
s(t, r, I, 0) & =\bar{\lambda} q^{e}(t, r, I), \quad \frac{\partial s(t, r, I, \epsilon)}{\partial \epsilon}=\underbrace{\frac{\partial \lambda(t, r, I, \epsilon)}{\partial \epsilon}}_{>0} q^{e}(t, r, I)>0, \\
\frac{\partial s(t, r, I, \epsilon)}{\partial I} & =\underbrace{\frac{\partial \lambda(t, r, I, \epsilon)}{\partial I}}_{<0} q^{e}(t, r, I)+\lambda(t, r, I, \epsilon) \underbrace{\frac{\partial q^{e}(t, r, I)}{\partial I}}_{<0}<0, \\
\frac{\partial s(t, r, I, \epsilon)}{\partial r} & =\underbrace{\frac{\partial \lambda(t, r, I, \epsilon)}{\partial r}}_{>0} q^{e}(t, r, I)+\lambda(t, r, I, \epsilon) \underbrace{\frac{\partial q^{e}(t, r, I)}{\partial r}}_{\geq 0}>0 .
\end{aligned}
$$

We note that given a concrete functional form for the intensity $\lambda$, and the loss quota distribution and, thus, $q^{e}$ the above effects on the short spread can actually be quantified. Unfortunately, this is not going to be the situation when dealing with forward credit spreads.

Before, however, we move on to forward credit spreads, we derive the dynamics by of the short credit spread under $\mathbb{Q}$.

Proposition 3.7. The dynamics of the short credit spread under $\mathbb{Q}$ is, in short hand notation, as follows

$$
\begin{aligned}
d \bar{s}= & \left(\frac{\partial s}{\partial t}+\frac{\partial s}{\partial r} a(t)+\frac{\partial s}{\partial I} \gamma\left(I_{t}\right) I_{t}+\frac{1}{2} \frac{\partial^{2} s}{\partial r^{2}} b^{2}(t)+\frac{1}{2} \frac{\partial^{2} s}{\partial I^{2}} \gamma^{2}\left(I_{t}\right) I_{t}^{2}+\frac{\partial^{2} s}{\partial I \partial r} b(t) \gamma\left(I_{t}\right) I_{t}\right) d t \\
& +\sigma_{s}(t) d W_{t}
\end{aligned}
$$

where

$$
\begin{aligned}
a(t) & =\left.\frac{\partial f(t, T)}{\partial T}\right|_{T=t}+\alpha(t, t) \\
b(t) & =\sigma(t, t) \\
\sigma_{s}(t) & =\frac{\partial s}{\partial r} b(t)+\frac{\partial s}{\partial I} \gamma\left(I_{t}\right) I_{t} .
\end{aligned}
$$

where $\alpha(t, T)$ and $\sigma(t, T)$ are the drift and the volatility of the forward risk-free interest rates in (2) and the usual HJM drift condition in (3) holds.

Proof. We apply Itô formula to equation (33).

In addition to the Itô formula we just need to deduce the dynamics of the risk-free short rate $r$, from the dynamics of the forward interest rate in (2). We recall $r(t)=f(t, t)$ and it is easy to show that 7

$$
d r(t)=\left(\left.\frac{\partial f(t, T)}{\partial T}\right|_{T=t}+\alpha(t, t)\right) d t+\sigma(t, t) d W_{t}
$$

\footnotetext{
${ }^{7}$ For a proof of this result see Björk (2004).
} 
Without a concrete functional form of the intensity and loss quota distribution, there is not much one can say about the dynamics of the short spread. Except, perhaps that since $\frac{\partial s}{\partial I}<0$ and $\frac{\partial s}{\partial r} \geq 0$ (recall Remark 3.6) we see that increases in the volatility of the index lead to decreases in the volatility of the short spread, while increases in the volatility of the short rate $r$ lead to increases in its volatility. Consequences to the drift cannot be drawn from Assumptions 3.2 and 3.3 alone.

\section{Remark 3.8. ( $\mathbb{P}$ considerations)}

The same qualitative relations would still hold under the objective probability measure $\mathbb{P}$ as long as the market price of jump risk is positive, which seems reasonable.

As to forward spreads $s(t, T)$, given that we could only obtain expressions in terms of expectations that have to be numerically evaluated, there little one can say.

In the next section we propose a way to model all the needed functions and show the simulation results.

\section{A concrete model}

In this section we illustrate the theoretical results previously derived using a "toy model".

Our purposes are that of highlighting the importance of considering the dependence between recovery and intensity of default, by showing that the results obtained are substantial, and of showing the applicability of our results, rather than that of being as realistic as we could.

For that reason, when setting up our model, we do it as simple as possible with the advantage of getting more tractable formulas and a better understanding about what is driving the simulation results.

The theoretical results apply, obviously, to the general case treated above and many more examples could have been considered.

In order to have a concrete model we need to:

- Establish the dependence of the volatility of the index $\gamma$, on the index level.

- Provide the intensity functional form for $\lambda$, in terms of $(t, r, I, \epsilon)$.

- Decide on a distribution for the loss quota $q$ for all possible $(t, r, I)$.

In our toy model we take the risk-free rate, $r$, as constant and abstain from considerations about the risk-free interest rates term structure. This is not realistic, but is also not harmful to our goal of understanding the impact of spreads.

$I$ is supposed to be the price of a traded asset. To consider non a non-traded asset, we would need further considerations in terms of the market price of index risk.

For simplicity we also take all functions to be time homogenous, the extention to non time homogeneous functions is straightforward.

Given these simplifications, to have a completely specified model to simulate we need to we define a function $\gamma(I)$ for the index volatility, a function $\lambda(I, \epsilon)$ for the intensity and, a distribution function $K(d q, I)$ for the loss quota. 


\subsection{Choosing the market index volatility $\gamma$}

We start by defining a concrete volatility for the index $\gamma(I)$. As stated in Assumption 3.1 we would like to the index volatility inversely related to the index level.

We start by defining a ratio which relates the current value of the index to its long-run trend value. Let us define

$$
m(I)=\frac{\bar{I}}{I}
$$

where $\bar{I}$ is a priori given and can be interpreted as the long-run trend value of the market index.

The above ratio measures how close or far away from the long-run trend value parameter, $\bar{I}$, the current value of the index $I$ is. Intuitively, it seems reasonable to make the volatility dependent on some relative value of the index, instead on its absolute value. $\bar{I}$ will be assumed to grow at the risk-free rate over time.

Reasonable levels for $m(I)$ typically range from 0.7 and 1.3. We note that the higher the current level of the index the lower is $m(I)$, i.e.

$$
\frac{\partial m}{\partial I}=-\frac{\bar{I}}{I^{2}}<0
$$

That is, a value of, say, $m=0.7$ refers to a bull market while $m=1.3$ refers to a bear market.

Based on the above ratio we now define the volatility of index as a function of our moneyness level $m$ in the following way

$$
\gamma(I)=\bar{\gamma}(m(I))^{\frac{1}{2}} \quad \forall I, \bar{\gamma} \in \mathbb{R}_{+} .
$$

In accordance with Assumption 3.1, the higher the current value of the index the lower is the index volatility $\gamma$

$$
\frac{\partial \gamma(I)}{\partial I}=\underbrace{\bar{\gamma} \frac{1}{2}}_{>0} \underbrace{[m(I)]^{-\frac{1}{2}}}_{>0} \underbrace{\frac{\partial m(I)}{\partial I}}_{<0}<0 \quad \forall I>0 .
$$

Figure 1 shows us two possible paths for the index process, one assuming $\gamma$ to be just a constant and the other where the index volatility depends on the index level as in (35).

\subsection{Choosing default intensities and the loss quota distribution}

\subsubsection{Default intensity}

Having defined the index volatility we now define the intensity function

$$
\lambda(I, \epsilon)=\bar{\lambda}[m(I)]^{\epsilon}=\frac{\bar{\lambda}}{\bar{\gamma}}[m(I)]^{\epsilon-\frac{1}{2}} \gamma(I) \quad \text { for } \bar{\lambda} \in \mathbb{R}+\text { and } \epsilon \in[0,1] .
$$

We note that with this modelization we can interpret the intensity function as a function of the index level or, if we prefer, as a function of the index volatility. One can argue that the intensity should not be affected by index level, but instead by its volatility since it is the volatility that 


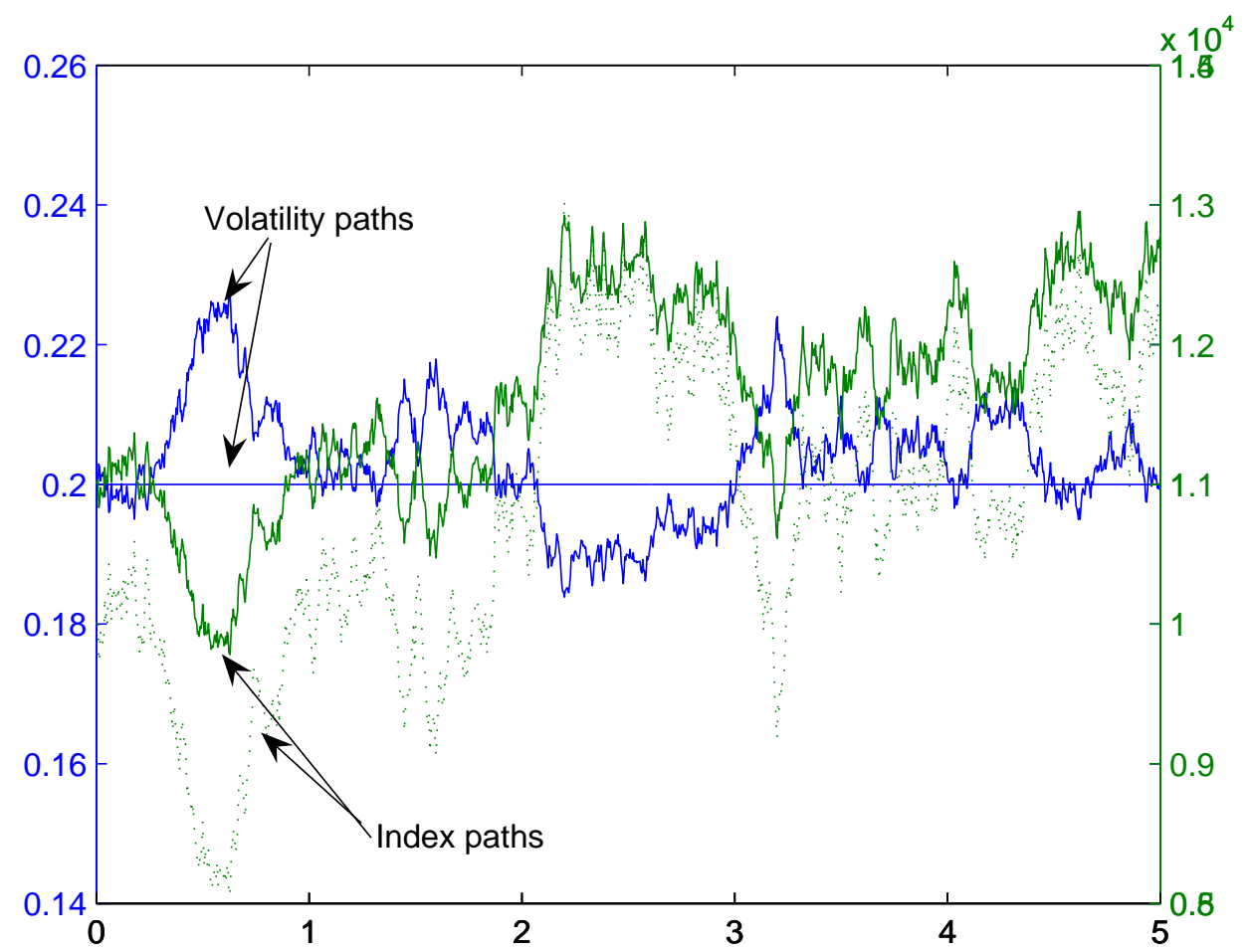

Figure 1: Two paths for the index level and volatility. The same noise was used for both cases, and we took $\bar{I}=10000$ and $I_{0}=10000$. Case 1 : constant volatility $\gamma=0.2$, the index process is the full line. Case 2: stochastic volatility as in (35), the index process is the dotted line.

represents the "risk". The above definition includes the two possibilities.

$$
\begin{aligned}
& \frac{\partial \lambda}{\partial I}=\underbrace{\bar{\lambda} \epsilon}_{>0} \underbrace{(m(I))^{\epsilon-1}}_{>0} \underbrace{\frac{\partial m(I)}{\partial I}}_{<0}<0 \\
& \frac{\partial \lambda}{\partial \gamma}=\underbrace{\frac{\bar{\gamma}}{\bar{\gamma}}}_{>0} \underbrace{[m(I)]^{\epsilon-\frac{1}{2}}}_{>0}>0 .
\end{aligned}
$$

Figure 2 show the functions $\lambda(I)$ and $\gamma(I)$ for different values of $m(I)$.

\subsubsection{Loss quota $q$}

Finally, we need to decide on our loss quota distribution.

As before we will make use of our $m$ ratio in setting up the distribution of the loss process which we will consider to belong to the Beta class. We start by recalling some basic properties of the beta distribution. 

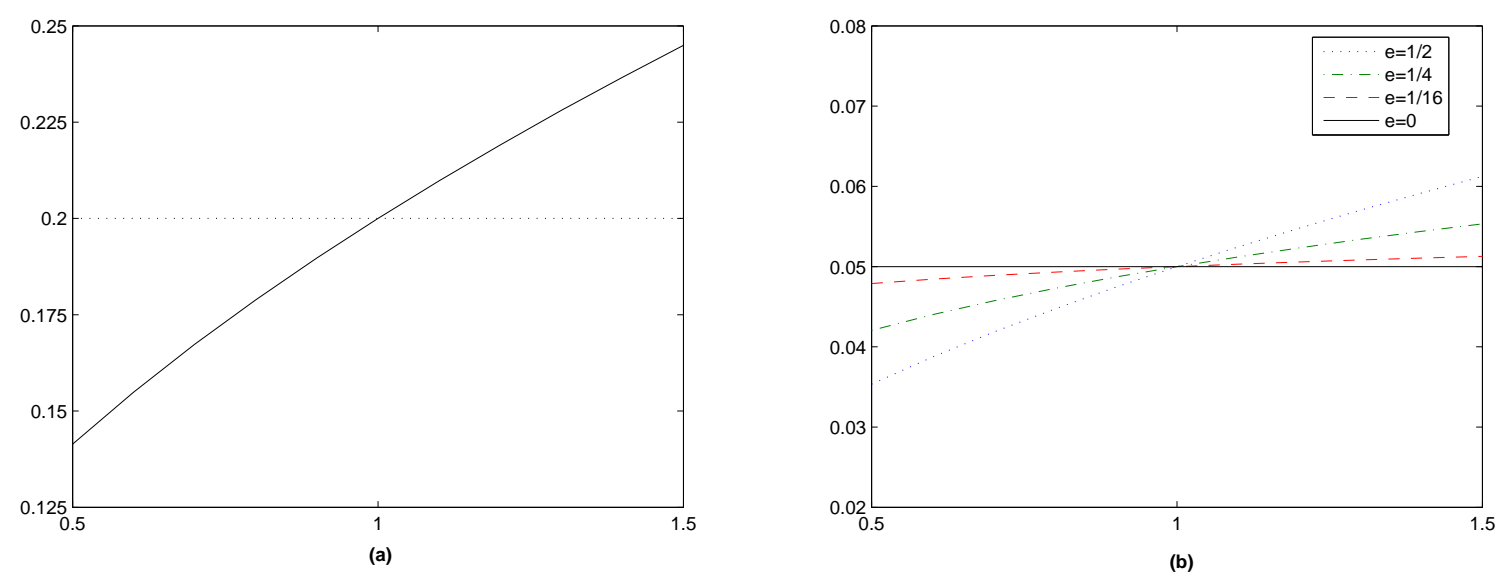

Figure 2: (a) : $\gamma(I)$ for different levels of $m(I)$ vs naive constant volatility $\bar{\gamma}=0.2$. (b): $\lambda(I)$, for different levels of $m(I)$ and different $\epsilon=0,1 / 16,1 / 4,1 / 2, \bar{\lambda}=0.05$.

Remark 4.1. The beta density function is given by

$$
f(x)=\frac{1}{B(a, b)} x^{a-1}(1-x)^{b-1} \mathbf{1}_{(0,1)}(x)
$$

where $a>0, b>0$ and $B(a, b)$ is the beta function:

$$
B(a, b) \int_{0}^{1} x^{a-1}(1-x)^{b-1} d s
$$

Furthermore, we have

$$
\mathbb{E}[X]=\frac{a}{a+b}=\mu \quad \operatorname{Var} X=\frac{a b}{(a+b+1)(a+b)^{2}} \quad \mathbb{E}\left[(X-\mu)^{r}\right]=\frac{B(r+a, b)}{B(a, b)}
$$

In our concrete application, for any fixed value of $I$, we choose

$$
q \sim \operatorname{Beta}(2 m(I), 2) \quad \text { i.e. } a=2 m(I) \text { and } b=2,
$$

which is consistent with the desired properties referred in Assumption 3.3.

Thus,

$$
\tilde{K}(q, I)=\frac{1}{B(2 m(I), 2)} \int_{0}^{q} x^{2 m(I)-1}(1-x) d x .
$$

Figure 3 shows us the loss quota density and its cumulative distribution function for three different values of $m(I): m=0.7$ representing a bull market, $m=1$ for the case where the market is at its long run level, and $m=1.3$ representing a bear market.

The next properties, follow from the properties of the Beta distribution:

- The expected loss is given by

$$
q^{e}(I)=\mathbb{E}[q(I)]=\frac{m(I)}{1+m(I)}
$$



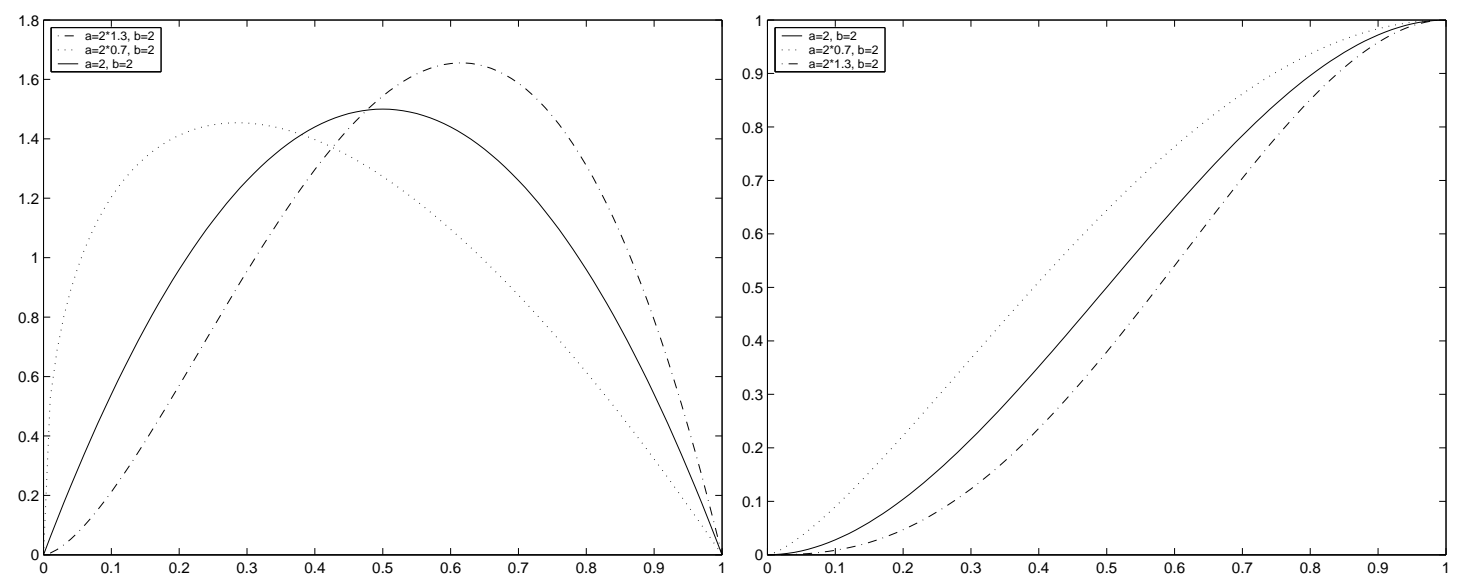

Figure 3: Density and Cumulative distribution functions of loss quota for $m=1.3, m=1, m=0.7$

$$
\text { - } \frac{\partial q^{e}(I)}{\partial I}=\frac{\frac{\partial m(I)}{\partial I}}{(1+m(I))^{2}}<0
$$

Furthermore

- if default occurs exactly at the long-run level the loss expected quota is exactly $1 / 2$;

- if default occurs when the index level is "high" $(m<1)$ one expects to recover more, expected loss quota decreases;

- if default occurs when the index level is "low" $(m>1)$ one expects to recover less, expected loss quota increases.

Figure 4 bellow shows both possible realizations of the loss quota (drawn from the beta density with the appropriate mean for each $m$ ) (stars), the expected loss quota levels for different values of $m$ (full line) in contrast with the naive approach of taking $\bar{q}=\frac{1}{2}$ (dotted line).

Before we go on we illustrate as well a possible relation between the intensity and the recovery process $(1-q)$ and the intensity $\lambda$. Figure 5 shows the scatter plot $\lambda$ versus one possible recovery realization for different levels of the index.

\subsection{Simulation Results}

In our simulations we use the Monte Carlo method where the step size is equivalent to one trading day (we do 250 steps per year) and all simulations concern 5000 paths. The same noise matrix is used for all scenarios and cases so that the values obtained can actually be compared (discretization errors would be in the same direction for all scenarios).

The spreads with zero maturity correspond to the short spread, for all other maturities correspond to the forward spread.

Table 1 let us the reference parameters, while Table 2 characterize all possible scenarios. 


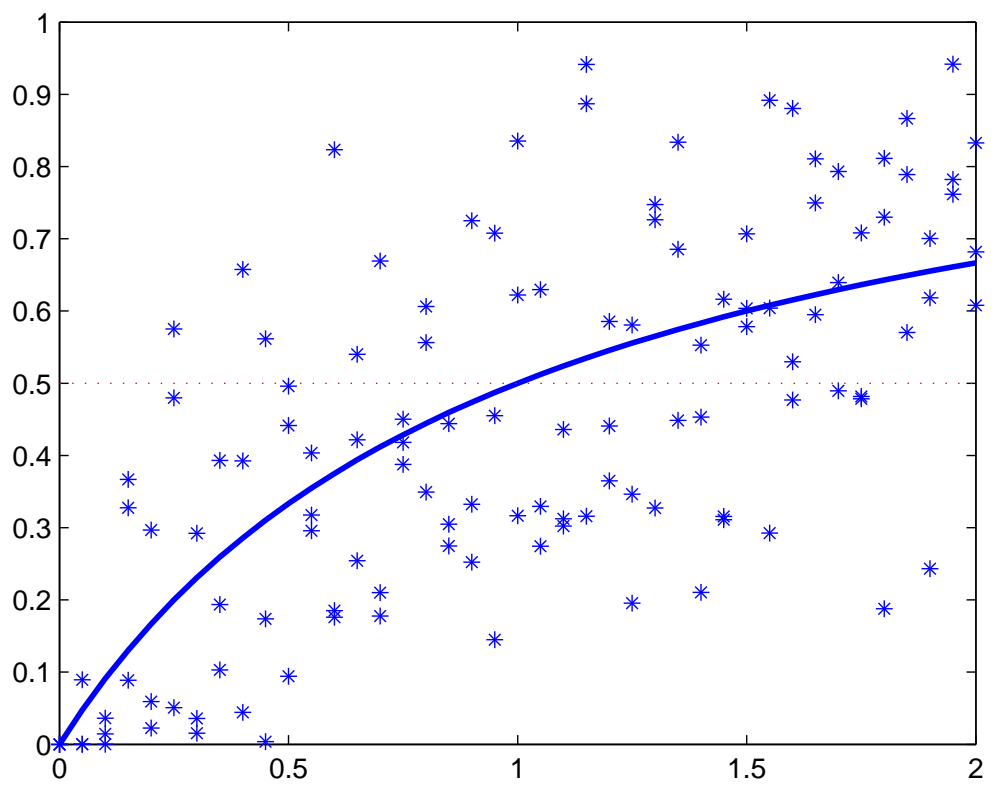

Figure 4: Loss quota possible realizations and expected value for different values of the "moneyness" $m$. Dotted line is the naive $q=\frac{1}{2}$.

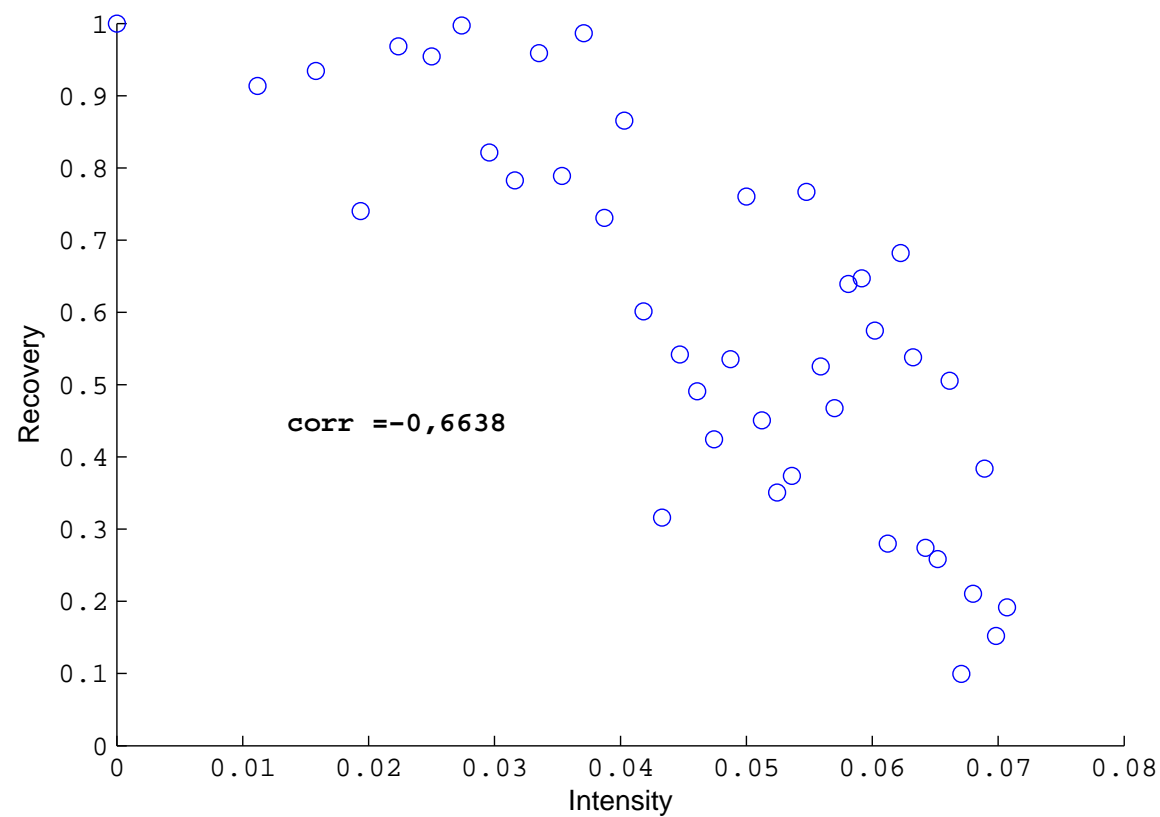

Figure 5: Scatter plot of intensity versus possible recovery realization for different values of $m$. 


\section{REFERENCE PARAMETERS}

\begin{tabular}{|c|c|c|}
\hline Maturities $(T)$ & \multicolumn{2}{|l|}{ From days up to 5 years } \\
\hline Risk-free interest rate & $5 \%$ & \\
\hline & Case A: bull market & 0.7 \\
\hline$m(I)$ & Case B: normal market & 1.0 \\
\hline & Case C: bear market & 1.3 \\
\hline Long-run index value & $10.000 e^{0.5 * T}$ & \\
\hline Fixed index volatility $(\bar{\gamma})$ & $20 \%$ & \\
\hline Fixed intensity value $(\bar{\lambda})$ & $5 \%$ & \\
\hline Fixed recovery value $\left(\bar{q}=\frac{1}{2}\right)$ & $50 \%$ & \\
\hline
\end{tabular}

Table 1: Reference values for the parameters in the model.

\section{DIFFERENT SCENARIOS}

\begin{tabular}{cccc}
\hline & & & \\
Scenario & Index Volatility & Intensity & Recovery \\
$(1)$ & $\mathrm{F}$ & $\mathrm{F}$ & $\mathrm{F}$ \\
$(2)$ & $\mathrm{S}$ & $\mathrm{F}$ & $\mathrm{F}$ \\
$(3)$ & $\mathrm{F}$ & $\mathrm{F}$ & $\mathrm{S}$ \\
$(4)$ & $\mathrm{S}$ & $\mathrm{F}$ & $\mathrm{S}$ \\
$(5)$ & $\mathrm{F}$ & $\mathrm{S}$ & $\mathrm{F}$ \\
$(6)$ & $\mathrm{S}$ & $\mathrm{S}$ & $\mathrm{F}$ \\
$(7)$ & $\mathrm{F}$ & $\mathrm{S}$ & $\mathrm{S}$ \\
$(8)$ & $\mathrm{S}$ & $\mathrm{S}$ & $\mathrm{S}$ \\
\hline
\end{tabular}

Table 2: Basic reference scenarios for simulations. $\mathrm{F}=$ Fixed, $\mathrm{S}=$ Stochastic.

\subsubsection{Credit spreads}

We start by looking into short spread dynamics.

Figure 6 presents three possible paths for the short spread under each scenario. Obviously, three paths are not representative in any sense, still we belive the intuition is nice and we chose paths with different characteristics. In $(a)$ the market index decreases over time, leading to an increase of the short spreads. In $(b)$ we have a mixed path and in $(c)$ the index value ends up increasing leading to a reduction in the short spreads. From the analysis of this figure, we can conclude that allowing for some stochasticity either in the intensity process or in the expected loss quota lead to similar short spread dynamics and that it is the combined effect that really makes the difference.

In any of the presented paths, if just one of the effects would be considered, the short spreads do not oscillate more than $1 \%$ bellow or above the naive $2.5 \%$, while for the combined effect the variation can be as large as $4 \%$ (in the case of path $(a)$ and quite often above $2 \%$.

Figure 7 give us the terms structure of credit spreads for three possible market situation: a bull market (where we took $m=0.7$ for the initial point), a normal market (initially $m=1$ ) and a bear market (at the beginning $m=1.3$ ). 
SHORT SPREAD DYNAMICS

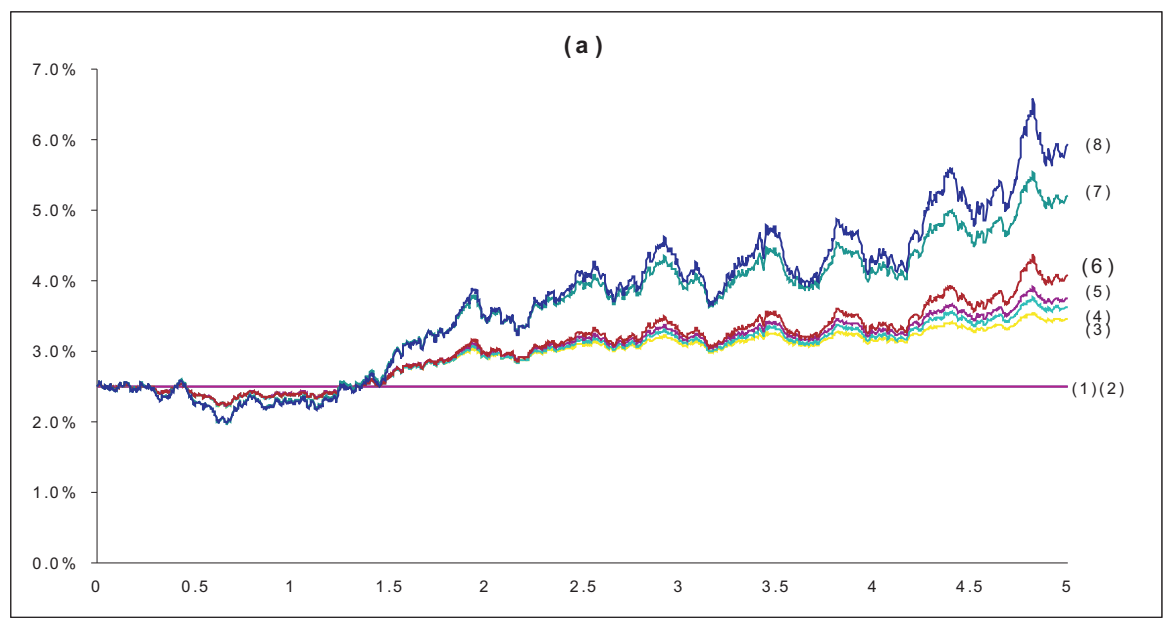

(b)

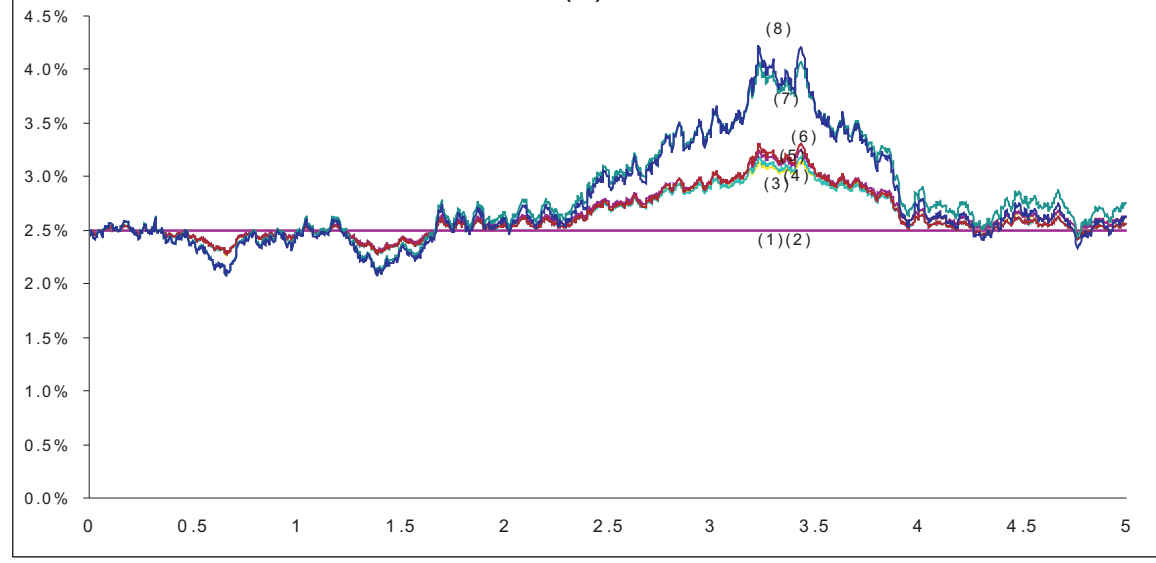

(c)

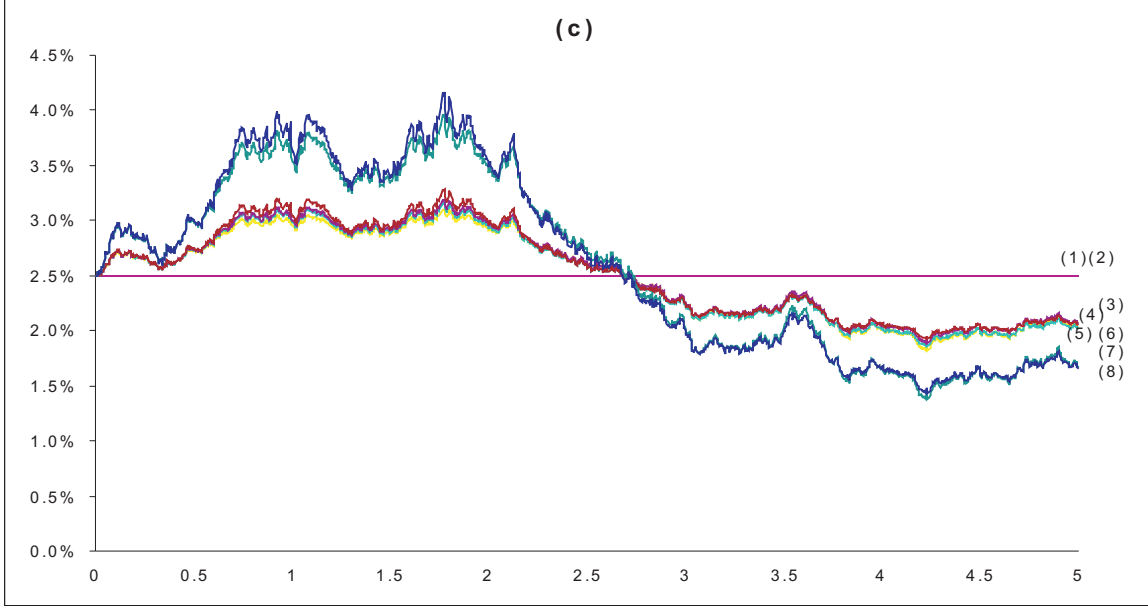

Figure 6: Three possible paths for the short spread, $s(t)$, dynamics. 
As expected, as long as the market index is allowed to have any influence either on the PDs or on the LGD the forward spread TS is not flat. It naturally increases with the maturity and its level is lower than $2.5 \%$ in bull markets and higher in bear markets. As in Figure 6 the effect of the index on the intensity alone or on recovery alone is of the same order of magnitude. The intensity seems, however, to have a more pronounced effect on the slope over time. Nonetheless, the big difference results from the combined effect, specially when to that we associate the impact of the negative relation between the index level and its volatility. Note that scenario (8) give us the highest or the lowest term structure in all circumstances.

Table 3 give us some figures for these spreads at some maturities.

\subsubsection{Prices of credit securities}

The pricing of many credit derivatives can be made by computing what is know as key building blocks. See for instance Schönbucher (2003) or Gaspar and Schmidt (2005).

Those building blocks are:

- The price of a zero-coupon defaultable bond with maturity $t$, under zero recovery,

$$
\bar{p}_{o}(t, T)=\mathbb{E}_{t}^{\mathbb{Q}}\left[e^{-\int_{t}^{T} r(u)+\lambda(u) d u}\right] .
$$

- The price at time $t$ of a zero-coupon defaultable bonds with maturity $T$ under non-zero recovery, in our case,

$$
\bar{p}(t, T)=\mathbb{E}_{t}^{\mathbb{Q}}\left[e^{-\int_{t}^{T} r(u)+\lambda(u) q^{e}(u) d u}\right]
$$

- The implied survival probabilities during the interval $[t, T]$

$$
\operatorname{prob}(t, T)=\mathbb{E}_{t}^{\mathbb{Q}}\left[e^{-\int_{t}^{T} r(u)+\lambda(u) q^{e}(u) d u}\right]
$$

- The price of a default digital payoff of 1 at default time, if default occurs in the interval $[t, T]$

$$
\text { digital }=\int_{t}^{T} e(t, s) d s
$$

where

$$
e(t, T)=\mathbb{E}_{t}^{\mathbb{Q}}\left[\lambda(T) e^{-\int_{t}^{T} r(u)+\lambda(u) d u}\right]
$$

Tables 3 (b) and 4 (a),(b),(c) show us the values of all these key quantities for various scenarios and different possible maturities.

Looking into Table 3 (b) the first thing that should be highlighted is that, even for low maturities there is a difference in the prices produced by naive scenarios $(1),(2)$, scenarios where just the PD or the LGD is depended on the index level ((3),(4) for the LGD and (5),(6) for the PD), and the scenarios were we consider the combined effect. We note that for the bull and bear market case the difference in pricing is already evident for the bonds with approximately one month to maturity $(T=0.1)$ and that with a maturity of 5 years the underpricing of the naive model can be of up to $5 \%$ in a bull market, and up to $10 \%$ in a bear market situation. In normal market situations the difference between the scenarios are slope differences with an overall tendency to overprice by the naive model if in reality the market index affects the PD, the LGD or both. 
FORWARD SPREADS
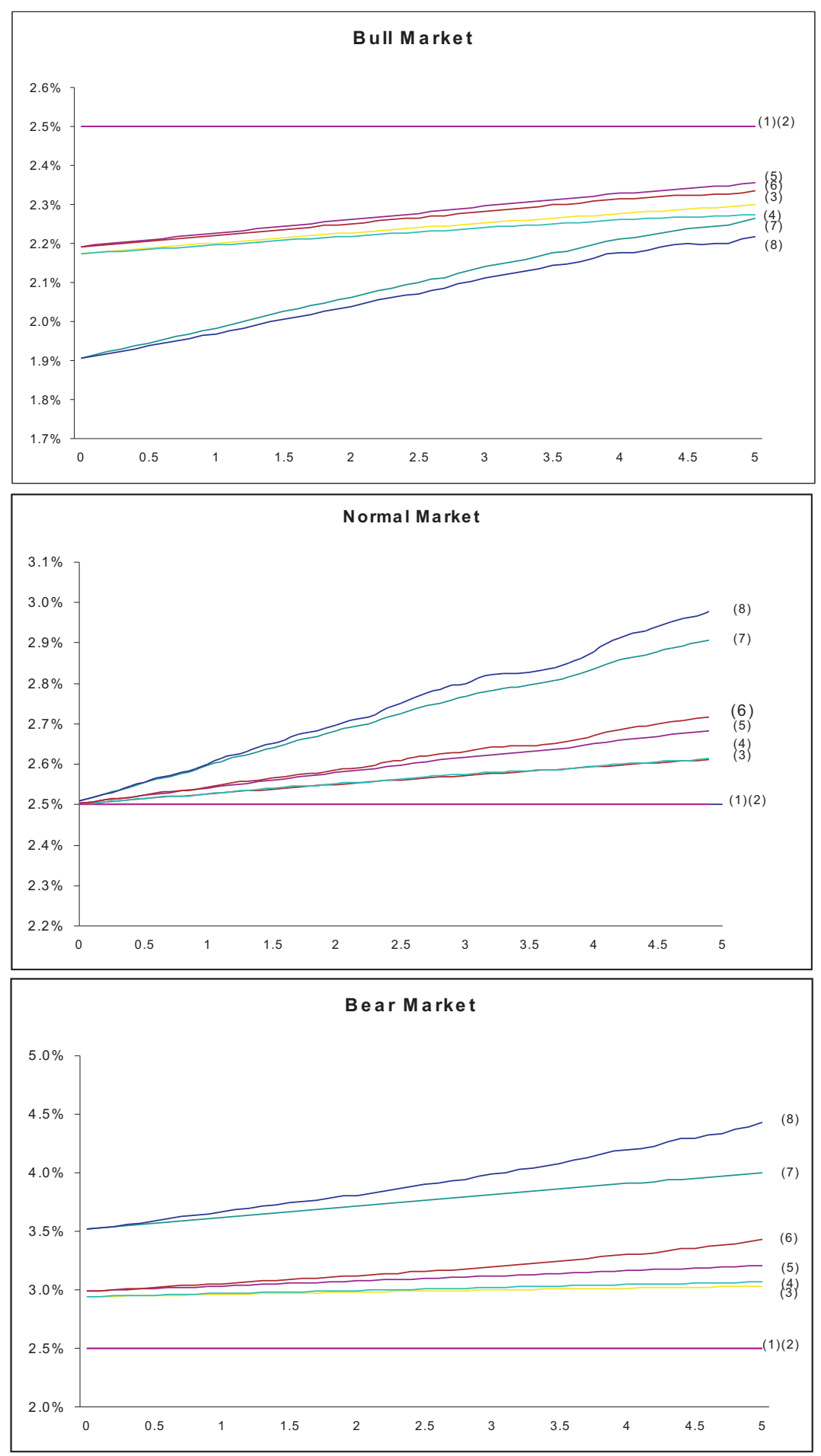

Figure 7: Forward spreads for all scenarios, under three possible market conditions. 


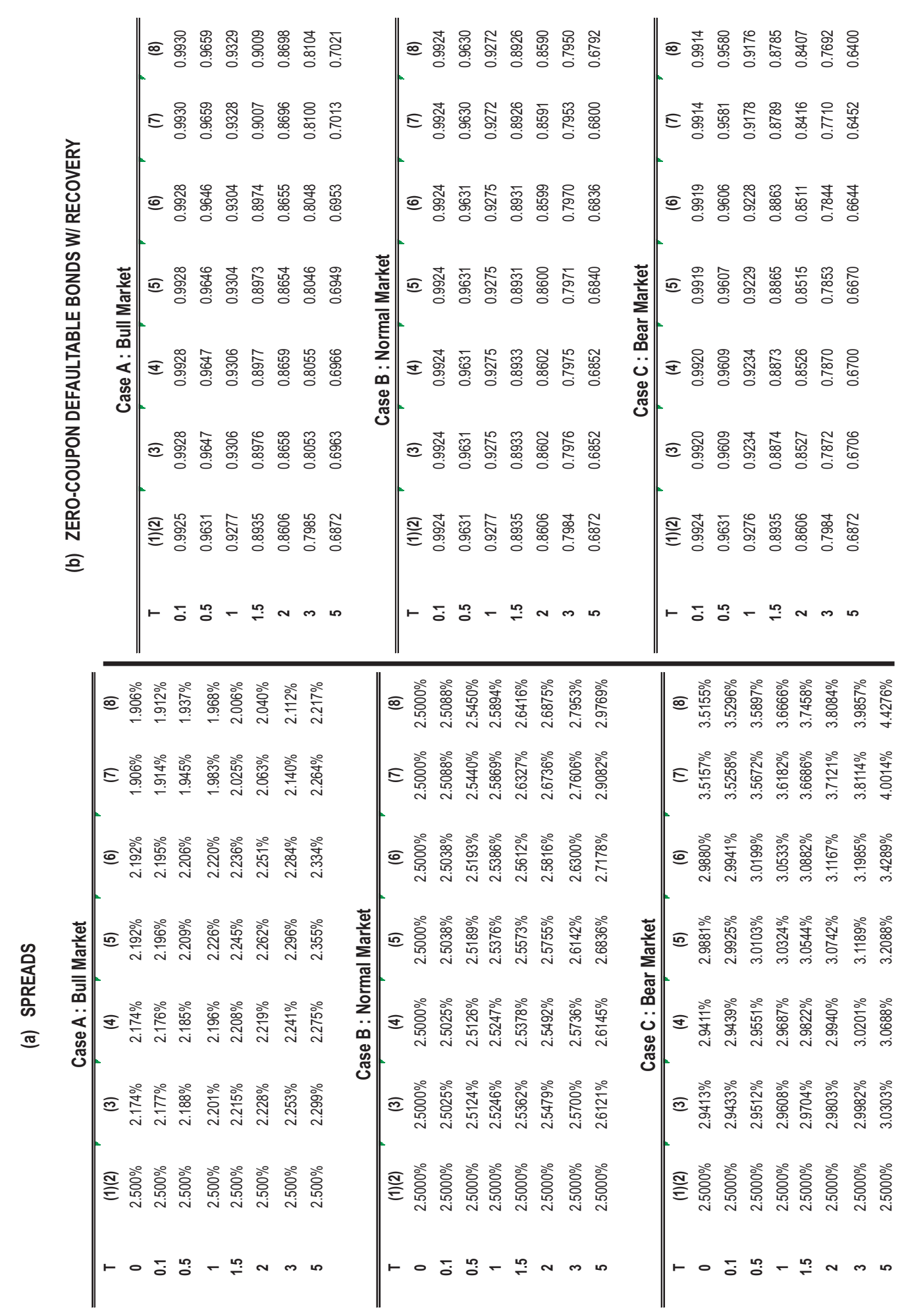

Table 3: Credit Spreads for several maturities $T=0,0.1,0.5,1,1.5,2,3,5$. For $T=0$ it is the short spread, for all others the forward spread. 

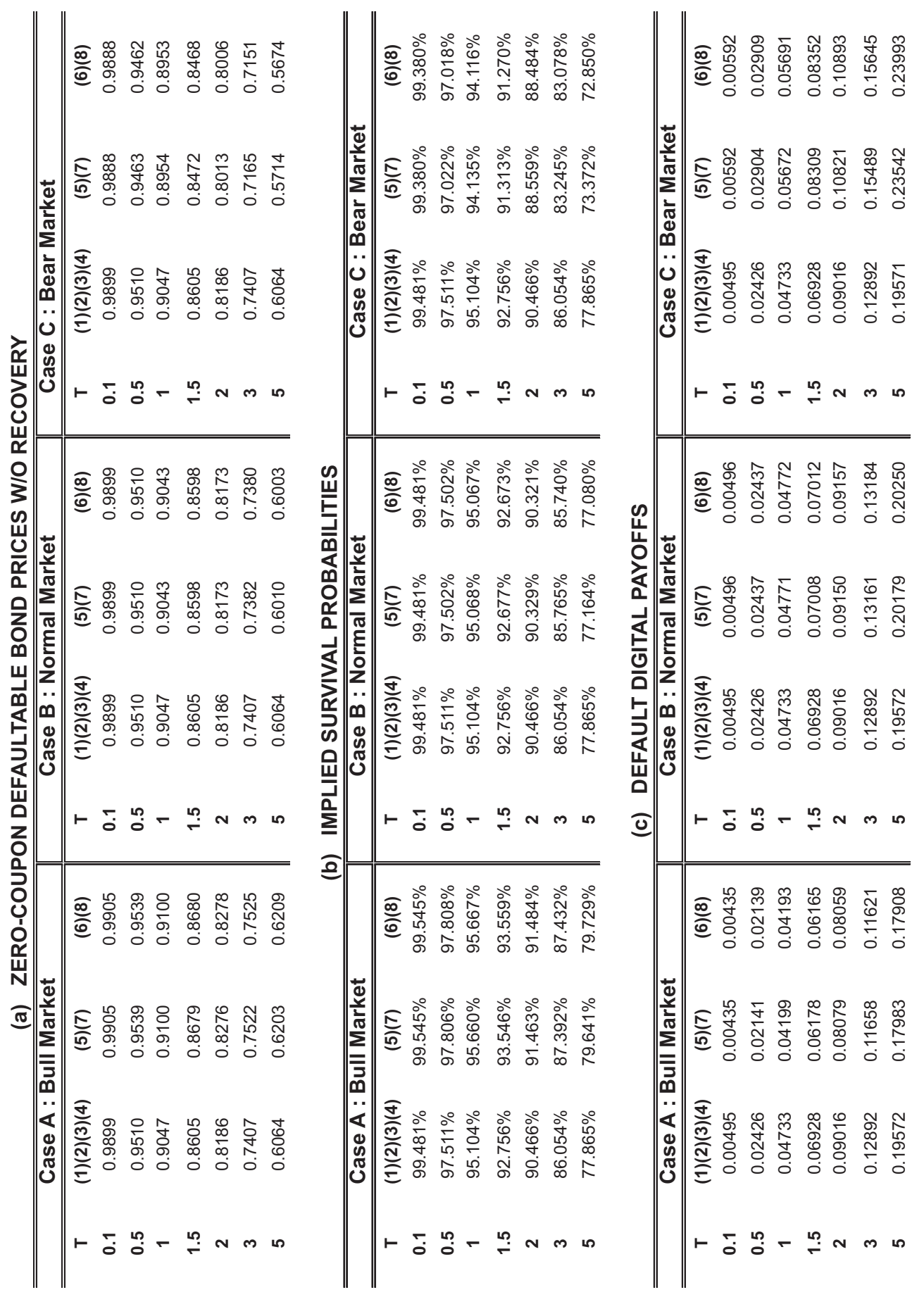

Table 4: (a) Prices of zero-recovery, zero-coupon bond prices for several maturities (top table). (b) Implied survival probabilities in the interval $[t, T]$ for several maturities. (c) Price of a digital payoff at default if it occurs in the interval $[t, T]$. Three different market conditions: bull, normal and bear. 
In Table 4 the quantities presented do not depend upon recovery (recall the building blocks equations), so all our scenarios reduce to three possibilities: constant intensity (case of scenarios $(1)(2)(3)(4))$, intensity dependent on the index that has or constant volatility (scenarios $(5)(7)$ ), or stochastic volatility (scenarios (6)(8)). In Table 4 (a), we see that the naive model can lead to underprice in bull markets also when we consider defaultable zero recovery bonds, and overpricing in bear markets. The dimension of these misspricings is similar to the misspricing in the previous table.

When looking into survival probabilities (Table4 (b)) the scenarios considering the index influence show less probability of surviving in bear markets than in bull markets, with a difference up to $7 \%$ already in the 5-year maturity. This indicates that using the naive model could lead to a possible overestimation of the true survival probabilities in bear markets and underestimate in bull markets. As in most of the previous tables, the dimension of the errors tend to be considerably bigger in bear markets than in bull markets. In the case of survival probability the overestimation can go up to $5 \%$ in bear markets in contrast with the up to $2 \%$ in bull markets. Finally, in Table 4 (c) we have the price if a digital payoff of 1 at default time. As before the differences to the naive model are more drastic in bear markets and can be considered quite drastic, specially, if one takes in consideration that an horizon of 5 years is quite short and all the impacts are likely to increase for higher maturities as we will see in the next section.

In Section 4.4 we run some maturity related robustness checks and draw the readers attention to what we consider to be interesting aspects related to the sensitivity parameter $\epsilon$ (assumed constant up to now) and possible tracking of credit spreads term structures by market volatilities.

\subsection{Additional Considerations}

\subsubsection{Higher maturities}

In this section we increase the maturity horizon we look at, and analyze to which extent our results hold. Table 5 presents forward spreads and defaultable bonds for several maturities, while Table 6 presents zero recovery bond prices implied survival probabilities and the price of default digital payoff of 1 . First of all we can clearly see that for all the three scenarios survival probabilities decrease significantly with the increase in the horizon $T$. If we increase the horizon from 5 to 15 years, in the stochastic volatility scenario the survival probability decreases almost by $40 \%$ for the bull market and up to $50 \%$ in case of the bear market. This is a realistic feature of our model in our opinion since at the longer time horizons when the market is in the recession and firms are known to be sensitive to the fluctuations of the market, the probability of default is quite high.

Moreover, it is also interesting to notice that although in case of the bull market stochastic volatility scenario yields higher survival probabilities at all the maturities, the difference in survival probabilities is much less at the higher maturities. For the case of the bear market, on the contrary, survival probabilities are lower for the stochastic volatility case, and the difference in the survival probabilities between the stochastic volatility and naive scenarios is more pronounced. In contrast to the bull market the difference increases by approximately $5 \%$ with the increase of the investment horizon from 5 to 15 years.

Overall the results are of the same nature as before with one interesting additional result for the long end of the forward spreads term structure. 
FORWARD SPREADS - Higher Maturities

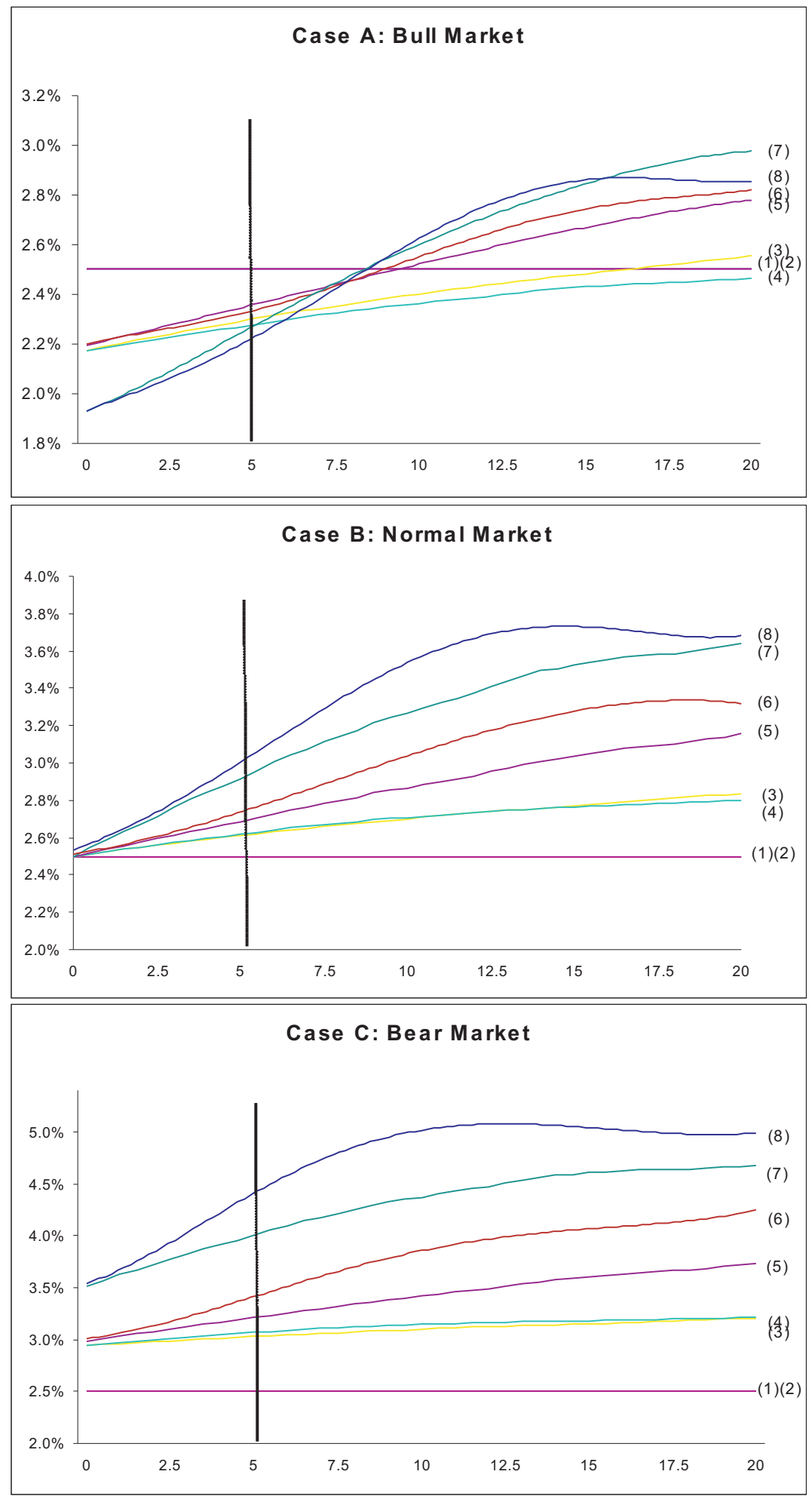

Figure 8: Forward spreads for all scenarios, under three possible market conditions and higher maturity values $T=5,6,7,8,10,12,15,10$. 
From Figure 8 we see that when we consider the dependence of both PD and LGD and the negative relation the index level and volatility (scenario (8)), the TS seems to converge faster to its long-run level. In fact, for maturities higher than 15 years the TS of this scenario are relatively flat. Thus, the forward credit spreads are most sensitive to the influence of the market index at the relatively shorter maturities, and somewhere at maturity of around 15 years the credit spreads become relatively more flat and less sensitive to the market index, moving in fact closer to each other.

\subsubsection{Ratings and different sensitivities}

We now look into the parameter $\epsilon$ in

$$
\lambda(I, \epsilon)=\bar{\lambda}[m(I)]^{\epsilon},
$$

which is a measure of the sensitivity of a firms PD to the market situation.

The intuition comes from the fact the PD of high credit worthiness firms should depend much less on market oscillation than that of less credit worthiness firms more sensitive to business cycles. In this sense different $\epsilon$ parameters could represent the term structure of firms with different rating levels.

In the following we consider three different values for epsilon: high $\epsilon=1 / 2$, medium $\epsilon=1 / 4$ and low $\epsilon=1 / 16 .^{8}$

Figure 9 and Tables 7 and 8 show the simulation results for the different $\epsilon$ values, under normal market conditions. The key feature that results from considering different sensitivities is that the TS of less sensitive firms have smaller TS slope. This is particularly obvious for scenarios (7)(8) when the index influence both PD and LGD and less obvious when it affects only one of them.

Thus, from a practical point of view it is more important to take into account the correlation with the market index when considering a portfolio of securities with low credit rating. We believe that the value of the portfolio will fluctuate significantly together with the business cycle. The effect will be even more pronounced for the low credit rating firms and especially in case of the stochastic volatility index specification.

\subsubsection{Using implied ATM volatilities as credit spread trackers}

An interesting side effect of our model, when we take the index volatility to be stochastic and negatively related to the index level, is that short spread dynamics can be quite well tracked by observing the index volatility. See Figure 10 with 3 possible volatility paths and compare to the short spread evolution for the same paths on Figure 6.

If the (spot) volatility seems to be a good tracker of the short spread, then implied volatilities of options with higher maturities may be good trackers of the forward spread TS. All this results from the negative correlation between the index level and its volatility.

This gives some fundamental reasoning for using implied volatilities of options on indices as predictors of the forward spread term structure and is in line with what seems to be real life common practice among traders who typically use at-the-money (ATM) volatility term structures in predicting forward credit spreads. For example, Collin-Dufresne, Goldstein, and

\footnotetext{
${ }^{8}$ The case of total insensitivity, or $\epsilon=0$, is always considered since in scenarios $(1)(2)(3)(4) \lambda(I, \epsilon)=\bar{\lambda}$.
} 


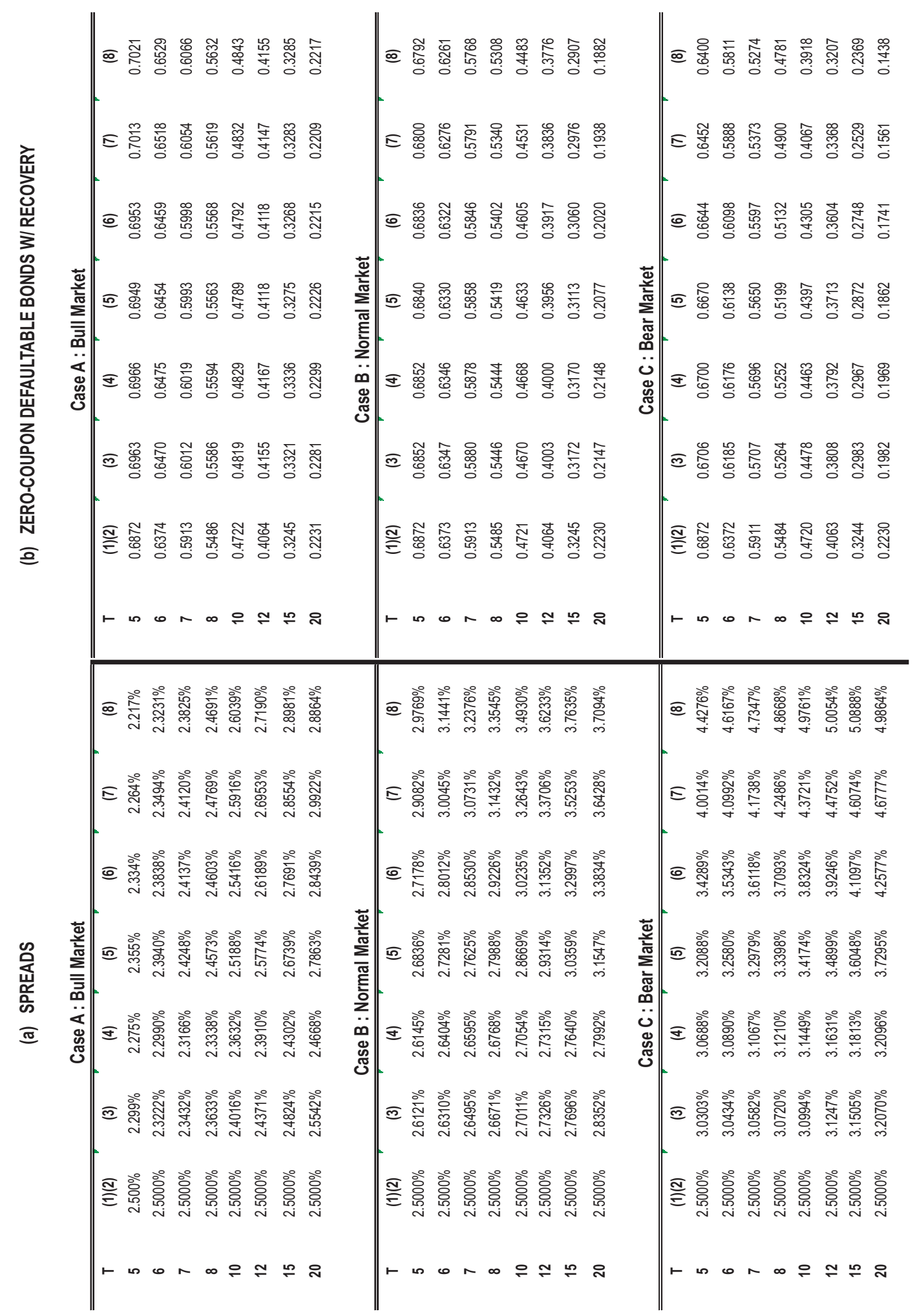

Table 5: (a) Credit Spreads and (b)Price of defaultable bond with recovery for several higher maturities $T=5,6,7,8,10,12,15,20$. 

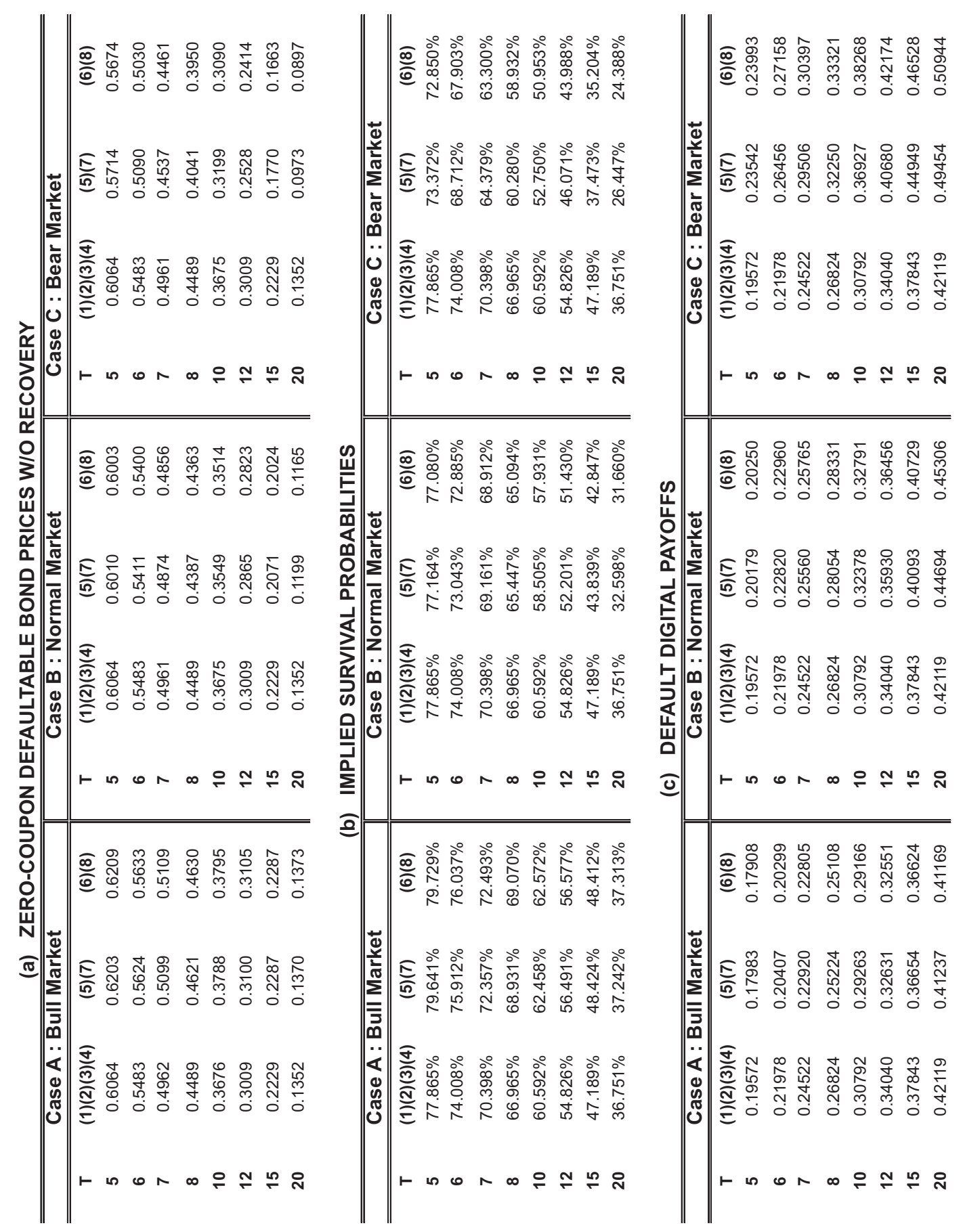

Table 6: (a) Prices of zero-recovery, zero-coupon bond prices for several maturities (top table). (b)Implied survival probabilities in the interval $[t, T]$ for several maturities. (c) Price of a digital payoff at default if it occurs in the interval $[t, T]$. 
FORWARD SPREADS - Different market Sensitivities
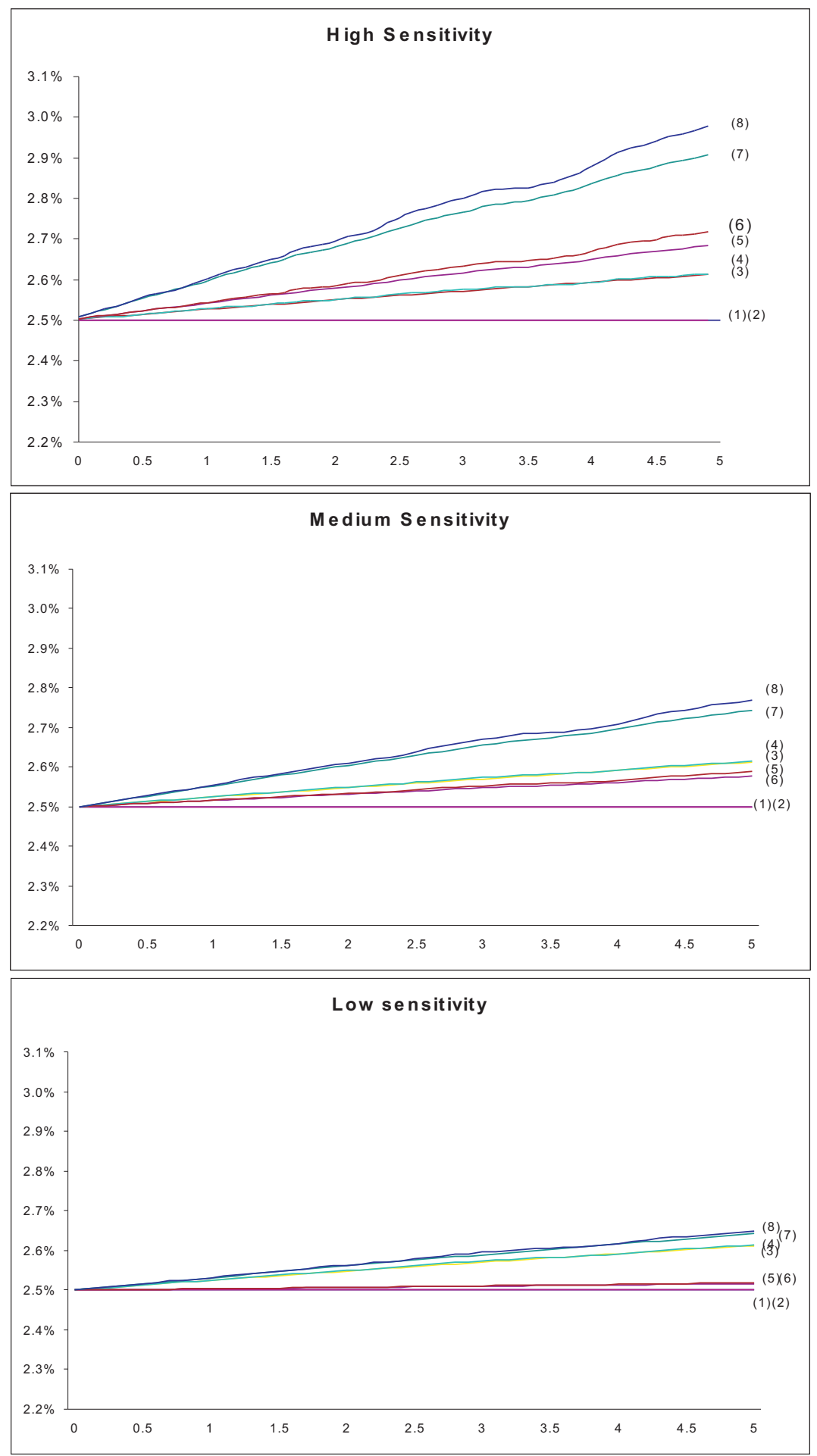

Figure 9: Forward spreads for all scenarios, under normal market conditions. For three different values of $\epsilon$ : high $\epsilon=\frac{1}{2}$, medium $\epsilon=\frac{1}{4}$ and low $\epsilon=\frac{1}{16}$. 


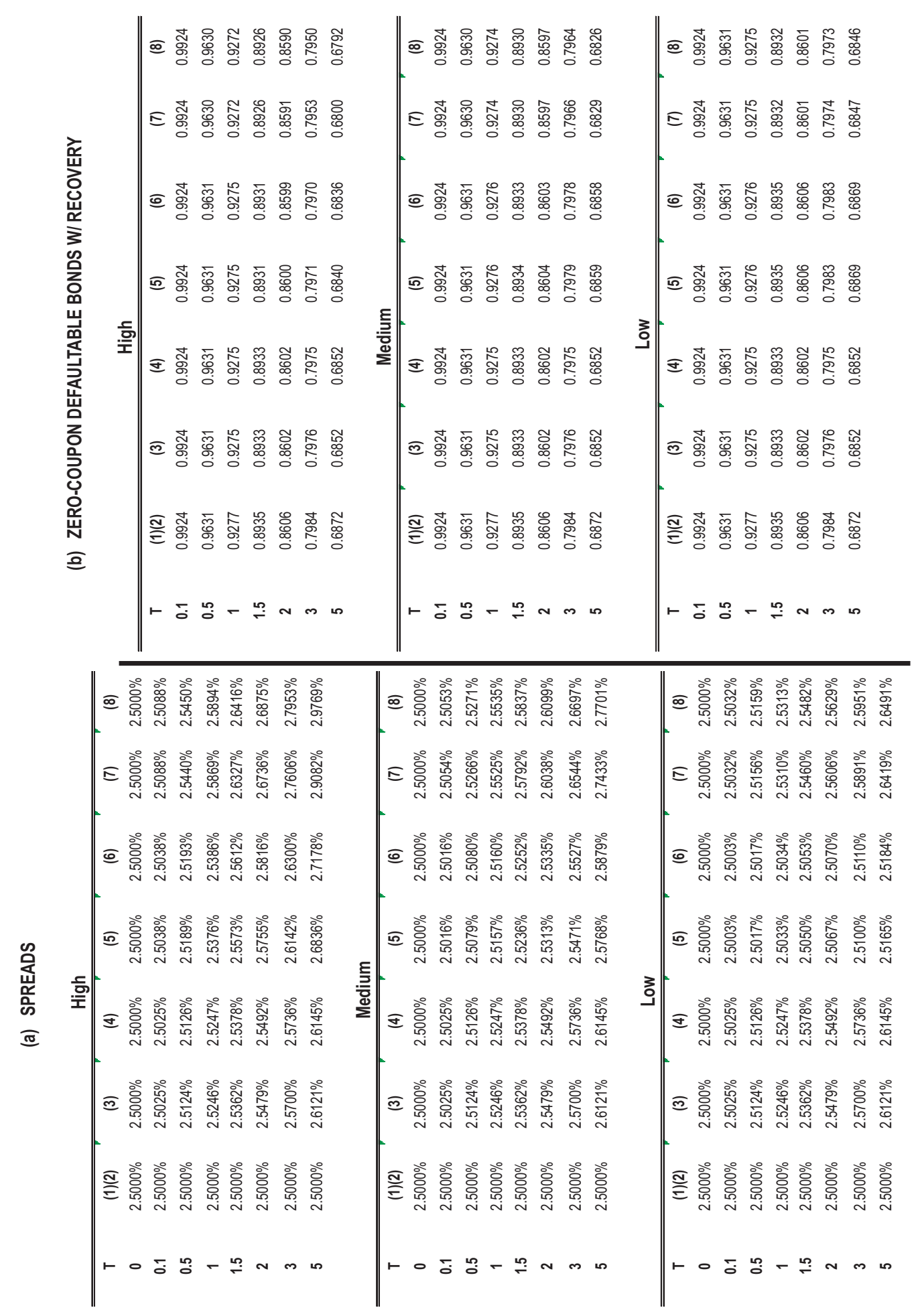

Table 7: (a) Credit Spreads and (b)Price of defaultable bond with recovery for several maturities and three different values of $\epsilon$ : high $\epsilon=\frac{1}{2}$, medium $\epsilon=\frac{1}{4}$ and low $\epsilon=\frac{1}{16}$. 


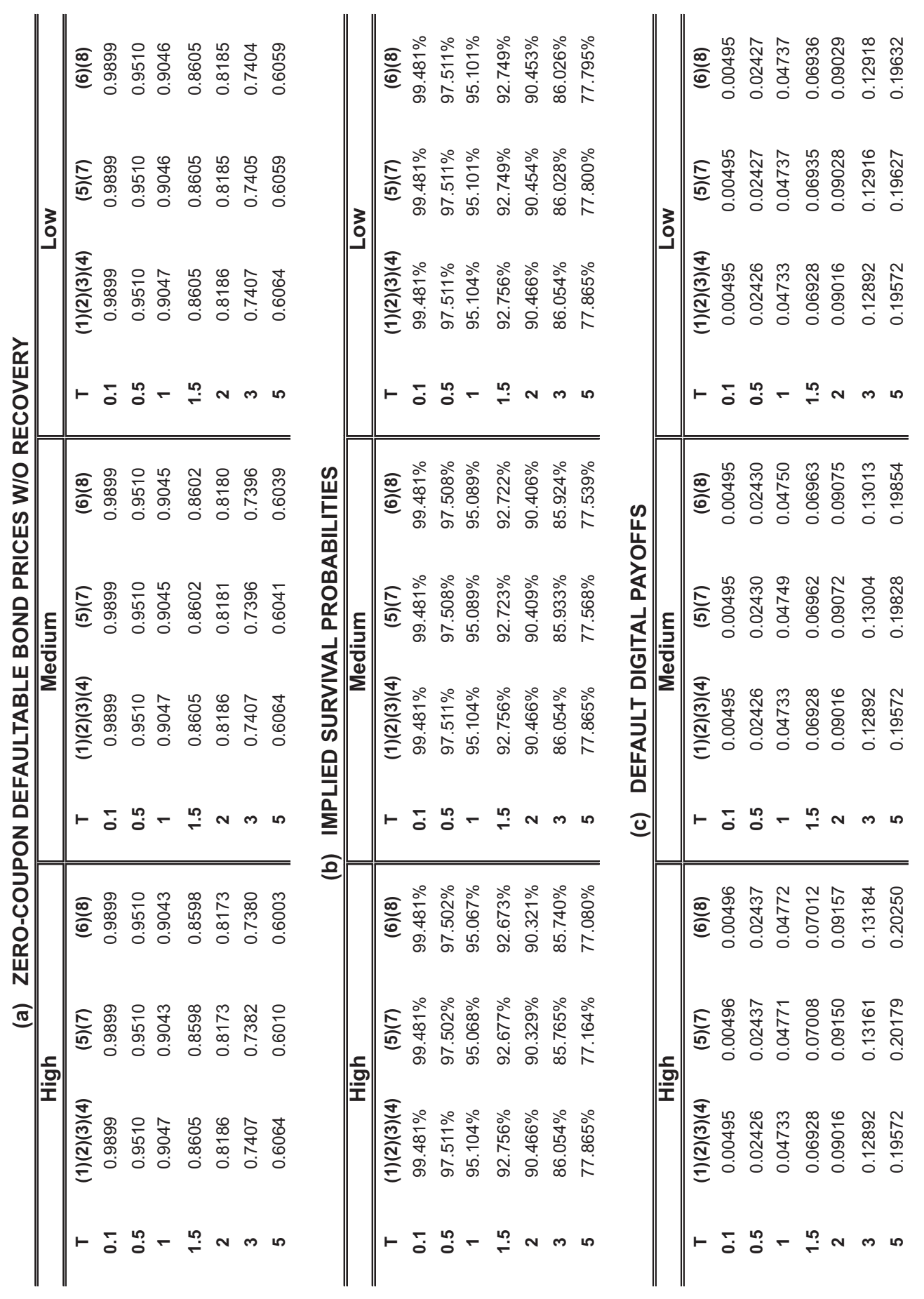

Table 8: (a) Prices of zero-recovery, zero-coupon bond prices for several maturities (top table). (b)Implied survival probabilities in the interval $[t, T]$ for several maturities. (c) Price of a digital payoff at default if it occurs in the interval $[t, T]$. Three different values of $\epsilon$ : high $\epsilon=\frac{1}{2}$, medium $\epsilon=\frac{1}{4}$ and low $\epsilon=\frac{1}{16}$. Higher maturities and three different market conditions: bull, normal and bear. 


\section{VOLATILITY TRACKERS}

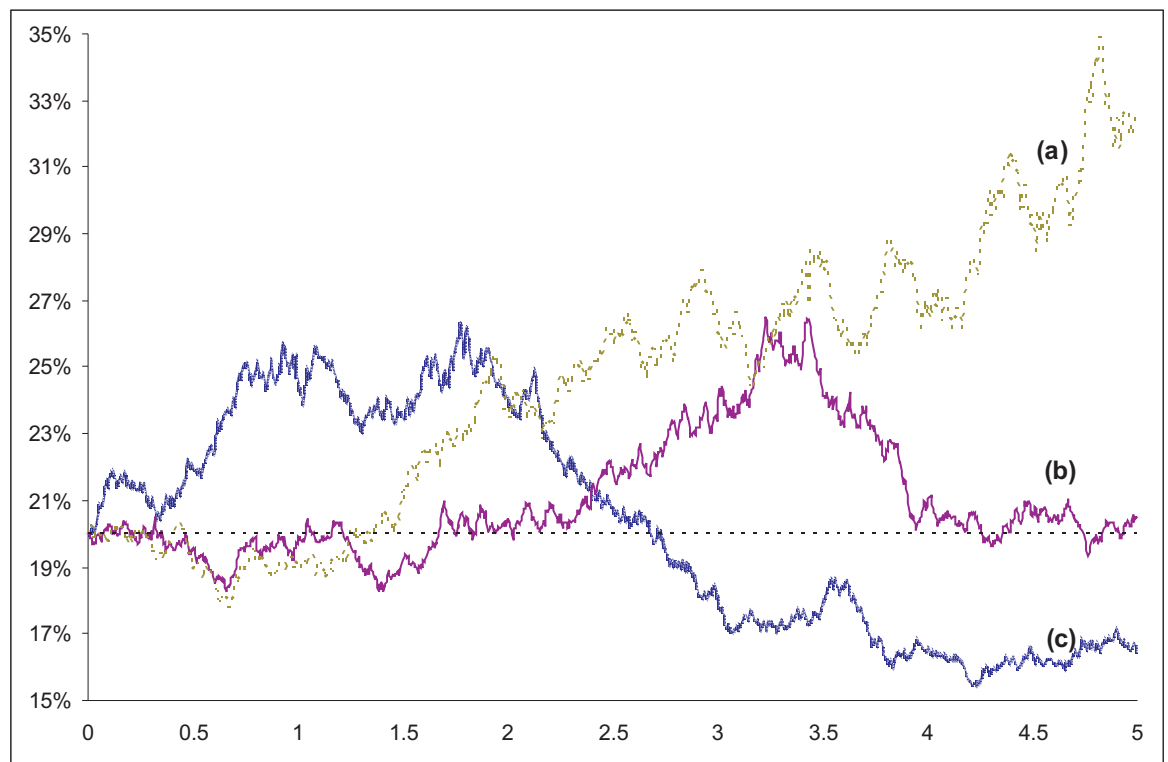

Figure 10: Volatility paths corresponding to the spot spread paths in Figure 6.

Martin (2001) investigated the determinants of credit spread changes. They showed that credit spreads are mostly driven by a single common factor and showed that implied volatilities of index options contain important information for credit spreads ${ }^{9}$.

\section{Conclusions and future research}

We propose a concrete reduced-form model where both the PD and LGD are dependent on a macroeconomic index. Furthermore, during depressions both the PD and the LGD increase (the reverse happens during booms). Finally, depressions are periods of higher market volatility, whiles booms are periods with low volatility.

By simulation we show that many of the realistic features of our model depend upon:

(i) the fact that both PD and LGD are driven by the same factors,

(ii) the negative relation of the index level and its volatility.

These two aspects together, unfortunately, make the model untractable, from an analytical point of view. Still this may be the right price to pay given all the behaviors it captures. The main realistic features captured by our model can be summarized as follows:

\footnotetext{
${ }^{9}$ Recent papers (see e.g. Cremers, Dreissen, and Weinbaum (2004)) start using measures of volatility and skewness that are based on individual stock options to explain credit spreads on corporate bonds. Implied volatilities of individual options are shown to contain important information for credit spreads. They showed that those implied volatilities improve on both implied volatilities of index options. However, in our framework we can not model this feature since the reduced models do not allow to model stock and corporate bonds together.
} 
- The difference between short spreads in bull versus bear markets can be up to three times more than the difference produced by models that consider the market influence in the PD or LGD only [mainly property (i)].

- Faster convergence to long-run levels, originating flat TS for maturities higher than 15 years [properties (i) and (ii)].

- Market volatility track quite well the short credit spread dynamics, suggesting that the TS of ATM implied volatilities of index options may do the same for forward credit spreads [mainly property (ii)].

It seems clear to us, that a model that has both characteristic (i) and (ii) will not allow for closed-form solutions. We would, nonetheless, like to finish the paper with a positive note related to future research.

Given the tractability drawback, and since one must rely on numerical simulations, the ideal would be to model the intensity and the distribution of the loss quota as realistic as possible (this may involve different functional forms and different market price of jump risk assumption). A study of the credit TS shapes observed in the market can be of help in defining such functional forms. Calibration of our "toy model" or other more complex model to market data seems to be another obvious next step. In addition to the single firm, this framework could also be extended to several firms and help dealing with portfolio credit risk issues. For portfolio credit risk the interdependence between PD and LGD is likely to have a much more relevant impact than at the individual firm level. Indeed, portfolio losses depend upon both quantities and the fact that periods when default is more likely may also be periods when recovery is lower, suggest caution is using naive models to establish bank reserves and related precautionary measures. Finally, our last comment on volatility trackers may help in constructing the bridge connecting the equity and credit markets and deserves being further investigated.

\section{References}

Altman, E. B., A. Resti, and A. Sironi (2004). Default recovery rates in credit risk modelling: a review of the literature and empirical evidence. Economic Notes by Banca Monte dei Paschi di Siena SpA 33(2), 183-308.

Basel Committee (2003). The new Basel capital Accord. Basel Comittee on Banking Supervision.

Björk, T. (2004). Arbitrage Theory in Continuous Time (2nd ed.). Oxford University Press.

Collin-Dufresne, P., R. S. Goldstein, and J. S. Martin (2001). Individual stock-option prices and credit spreads. Journal of Finance 56(6), 2177-2207.

Cremers, M., J. Dreissen, and D. Weinbaum (2004). The determinants of credit spread changes. Working paper.

Düllmann, K. and M. Trapp (2000). Systematic risk in recovery rates - an empirical analysis of us corporate credit expousures. Discussion Paper Serie 2: Banking and Financial Supervision.

Elton, E. and M. J. Gruber (2004). Factors affecting the valuation of corporate bonds. Journal of Business and Finance 28(11), 31-53.

Frye, J. (2000a). Collateral damage. Risk 13(4), 91-94. 
Frye, J. (2000b). Depressing recoveries. Risk Magazine November, 108-111.

Frye, J. (2003). A false sense of security. Risk 16(8), 63-67.

Gaspar, R. M. (2001). Sobre o efeito da correlação entre rendibilidade e volatilidade do activo subjacente na valorização de opções. Serie Moderna Finança n.25,Euronext.

Gaspar, R. M. and T. Schmidt (2005). Quadratic models for portfolio credit risk with shotnoise effects. Working Paper SSE.

Giese, G. (2005). The impact of PD/LGD correlations on credit risk capital. Risk Magazine 8(4), 79-84.

Gordy, M. (2000). A comparative anatomy of credit risk models. Journal of Banking and Finance 24, 119-149.

JP Morgan (1997). Creditmetrics. Technical Document.

Saunders, A. (1999). Credit Risk measurement. John Wiley \& Sons.

Schönbucher, P. J. (2001). Factors models: portfolio credit risk when defaults are correlated. Journal of Risk Finance 3(1), 31-53.

Schönbucher, P. J. (2003). Credit derivatives pricing models - models, pricing and implementation. JWS.

Wilde, T. (2001). IRB approach explained. Risk 14(5), 87-90.

Wilson, T. (1997). Portfolio credit risk. Risk 10(9-10), 111-117, 56-61. 